\title{
The role of gamma-synuclein in cocaine-induced behaviour in rats
}

\author{
Frederic Boyer and Jean-Luc Dreyer \\ Institute of Biochemistry, University of Fribourg, Rue du Musée 5, CH-1700 Fribourg, Switzerland
}

Keywords: addiction, cocaine, drugs of abuse, gamma-synuclein, in vivo gene transfer, lentivirus, siRNA

\begin{abstract}
The aim of this study was to investigate the role of $\gamma$-synuclein in the rewarding effects of chronic cocaine administration and its putative interaction with the dopamine transporter (DAT). For this purpose, regulatable lentiviruses driving overexpression of the rat $\gamma$-synuclein or DAT have been prepared, as well as lentiviruses expressing siRNAs, aimed at silencing either DAT or $\gamma$-synuclein mRNA expression. Overexpression of DAT in the nucleus accumbens (NAc) induced a $35 \%$ decrease in locomotor activity, which could be abolished when the same animal was fed doxycycline. Furthermore, local inhibition of DAT in the NAc, using lentiviruses expressing siRNAs targeted against DAT, resulted in significant hyperlocomotion activity (72\% increase over controls). By contrast, overexpression of $\gamma$-synuclein in the NAc alone had no effect, while local silencing lead to a significant decrease in cocaine-induced locomotor activity (47\% decrease compared with controls). Surprisingly, coinjection lentiviruses expressing DAT and $\gamma$-synuclein - leading to overexpression of both proteins in the NAc - resulted in a strong increase in cocaine-induced rat locomotor activity (52\% increase compared with controls), which was abolished upon locally silencing these genes, suggesting a synergetic role of both proteins, possibly mediated through a direct interaction.
\end{abstract}

\section{Introduction}

Cocaine is a major psychostimulant, which binds to the dopamine transporter (DAT) and blocks the synaptic reuptake of dopamine leading to a marked elevation of synaptic dopamine. This may be responsible for mediating behavioural and reinforcing effects that contribute to its abuse liability. The shuttling of DAT to and away from the plasma membrane is modified by the action of $\alpha$-synuclein, thereby changing the efficiency of dopamine uptake at the nerve terminals (Sidhu et al., 2004b), e.g. in the mesolimbic dopaminergic system, a critical pathway mediating addiction to psychostimulant and reinforcement. $\alpha$-Synuclein binds to the DAT and serotonin transporter, affecting their activities and their recruitment to the plasma membrane, an effect that can be modulated by $\gamma$-synuclein expression (Wersinger et al., 2003a,b, 2006; Wersinger \& Sidhu, 2005).

Synucleins are small proteins, mainly expressed in neurons. Little is known about their normal functions, although some evidence suggests a role on synaptic vesicular transport and synaptic plasticity (Murphy et al., 2000; Li et al. 2002). They play an important role as regulator in dopaminergic transmission (Abeliovich et al., 2000; Mosharov et al., 2006) and regulate the filamentous network that in turn influences the release of dopamine and glutamate neurotransmitters involved in the mood and motivation process. The family includes three known proteins: $\alpha$-synuclein, $\beta$-synuclein and $\gamma$-synuclein (Li et al., 2002). The role of $\alpha$-synuclein in the genesis of neurodegenerative disorders is well known, whereas the other members of the synuclein family are less characterized. $\alpha$ - and $\gamma$-Synuclein are involved in dopamine

Correspondence: Professor J.-L. Dreyer, as above.

E-mail: jean-luc.dreyer@unifr.ch homeostasis, as both proteins bind to tyrosine hydroxylase (Perez et al., 2002; Xu et al., 2002) and other dopamine synthesis genes, such as GTP cyclohydrolase (Sidhu et al., 2004a), downregulating their activities (Kuhn et al., 2007). $\gamma$-Synuclein is overexpressed in breast cancer cells, where it upregulates matrix proteases (Surgucheva et al., 2003; Jiang et al., 2004) and plays a principal role in neuronal plasticity in the population of neurons involved in an adapted integration of mood response to environmental challenges (Kuhn et al., 2007). It is also involved in cell migration and adhesion (Jia et al., 1999), in ocular pathologies (Surguchov et al. 2001a, 2001b; Maurage et al., 2003) and some forms of neurodegeneration (Galvin et al., 1999, 2000; Wang et al., 2004). In addition, $\gamma$-synuclein influences neurofilament network integrity in cultured sensory neurons (Buchman et al., 1998) and activates Elk-1, mitogen-activating kinase and several types of matrix metalloproteases (Surguchov et al., 2001a), thus playing a role in remodelling of extracellular matrix and modulating axonal architecture, which are central also in addiction.

In a previous study, we have described an overexpression of $\alpha$ - and mainly of $\gamma$-synuclein in the mesolimbic dopaminergic pathway upon cocaine (Brenz-Verca et al., 2003), suggesting a key role in regulating this brain circuit upon addiction. Because $\alpha$-synuclein strongly controls the expression of the DAT transporter at the plasma membrane, a process modulated by $\gamma$-synuclein, we aimed in the present study at investigating the role of $\gamma$-synuclein in the rewarding effects of chronic cocaine administration in relation to DAT expression. For this purpose, lentiviruses driving the expression of the rat DAT or of $\gamma$-synuclein or expressing siRNAs targeted against either DAT or $\gamma$-synuclein mRNA were constructed and served for in vivo gene transfer into the rat nucleus accumbens (NAc). The impact of $\gamma$-synuclein and DAT expression on chronic cocaine administration was assessed under different conditions, using locomotor activity 
measurements. Our data suggest a synergic role of both proteins, which could pass through a direct interaction.

\section{Materials and methods \\ Lentivirus constructions \\ Construction of lentivirus- $\gamma$-synuclein ( $L V-\gamma$ Syn)}

The rat $\gamma$-synuclein cDNA (GenBank accession no. 031688) was polymerase chain reaction (PCR) -amplified from cDNA of cocaineinjected rats using the following primers: 5'-AAG GAT CCA ATG GGT TAC CCA TAC GAC GTC CCC AGA CTA CGC TAT GGA CGT CTT CAA T-3' and 5'-CCG CTC GAG CGG ATG AGT AAG AGC AAA-3'. The forward primer contains a BamHI restriction site followed by the haemaglutinin epitope sequence (in bold) and the rat $5^{\prime}-\gamma$-synuclein cDNA specific sequence, the reverse primer contains the rat $3^{\prime}-\gamma$-synuclein cDNA-specific sequence, a stop codon and a $X h o I$ restriction site. The PCR product was digested with $B a m H I$ and XhoI and cloned into BamHI/XhoI restriction sites into pTK431 (Bahi et al., 2005), which expresses the gene of interest under control of a Tet-Off promotor. The green fluorescent protein (GFP) expression vector pTK433 (Bahi et al., 2004a,b) was used as a control vector.

\section{Construction of lentiviruses- $\gamma$-synuclein-siRNAs ( $L V-\gamma \operatorname{Syn}$-siRNAs)}

To silence $\gamma$-synuclein expression in vitro and in vivo, three targets were designed according to the $\gamma$-synuclein mRNA sequence. The following targets within the $\gamma$-synuclein sequence were selected, based on Hannon's design criterion (http://katahdin.cshl.org:9331/RNAi/ html/rnai.html): first target: bp 21-52; second target: bp 184-212; third target: bp 342-368. To each oligo, a XhoI restriction site was added at $3^{\prime}$ and a U6-3'-specific 10 mer at $5^{\prime}$. Using the pSilencer 1.0-U6 (Ambion, UK) as a template and a U6 promoter-specific forward primer containing $\mathrm{BamHI}$ restriction site (5'-GCG GAT CCC GCT CTA GAA CTA GTG C-3'), each siRNA target was added to the mouse U6 promoter by PCR, using the following PCR program: $120 \mathrm{~s}$ at $94{ }^{\circ} \mathrm{C}$ (initial denaturation) followed by 35 cycles $\left(45 \mathrm{~s}\right.$ at $94{ }^{\circ} \mathrm{C}$, $45 \mathrm{~s}$ at $64{ }^{\circ} \mathrm{C}$ and $45 \mathrm{~s}$ at $72{ }^{\circ} \mathrm{C}$ ) in $4 \%$ dimethyl sulphoxide (Sigma, Switzerland). The PCR product was digested with BamHI and XhoI, cloned into similar sites into pTK431, and sequenced to verify the integrity of each construct.

\section{Construction of lentivirus-DAT (LV-DAT)}

The DAT DNA (GenBank accession no. 012694) was PCR amplified from pCMV5rDAT as a template and the following primers: 5'-CCG TTA ACA TGG ACT ACA AAG ACG ATG ACG ATA AGC CAG TAA GAG CAA ATG C-3' and 5'-CCG CTC GAG CGG TTA CAG CAA CAG CCA GTG ACG-3'. The forward primer contains a HpaI restriction site followed by a FLAG epitope sequence (in bold) and the rat $5^{\prime}$ DAT cDNA specific sequence, the reverse primer contains the rat 3' DAT cDNA-specific sequence, a stop codon and a XhoI restriction site. The PCR product was digested with $\mathrm{HpaI}$ and $\mathrm{XhoI}$, and cloned into HpaI/XhoI restriction sites into pTK431 (Bahi et al., 2005), which expresses the gene of interest under control of a Tet-Off promotor. The GFP expression vector pTK433 (Bahi et al., 2004a,b) was used as a control vector.

\section{Construction of lentiviruses DAT-siRNAs (LV-siRNAs-DAT)}

To silence DAT expression in vitro and in vivo, three targets were designed according to the DAT mRNA sequence. The following targets within the DAT sequence were selected, based on Hannon's design criterion (http://katahdin.cshl.org:9331/RNAi/html/rnai.html): first target: bp 19-47; second target: bp 864-890; third target: bp1827-1854. To each oligo, a XhoI restriction site was added at $3^{\prime}$ and a U6-3'-specific $10 \mathrm{mer}$ at $5^{\prime}$. Using the pSilencer 1.0-U6 (Ambion, UK) as a template and a U6 promoter-specific forward primer containing BamHI restriction site (5'-GCG GAT CCC GCT CTA GAA CTA GTG C-3'), each siRNA target was added to the mouse U6 promoter by PCR, using the following PCR program: $120 \mathrm{~s}$ at $94{ }^{\circ} \mathrm{C}$ (initial denaturation) followed by 35 cycles $\left(45 \mathrm{~s}\right.$ at $94{ }^{\circ} \mathrm{C}$, $45 \mathrm{~s}$ at $64{ }^{\circ} \mathrm{C}$ and $45 \mathrm{~s}$ at $72{ }^{\circ} \mathrm{C}$ ) in $4 \%$ dimethyl sulphoxide (Sigma). The PCR product was digested with BamHI and XhoI, cloned into similar sites into pTK431, and sequenced to verify the integrity of each construct.

\section{Lentivirus production}

The vector plasmids (either pTK431- $\gamma$-synuclein, pTK431-DAT, pTK433-GFP, pTK431-U6- $\gamma$ Syn-siRNAs, pTK431-U6-DAT-siRNAs), together with the packaging construct plasmid $p \cdot N R F$ and the envelope plasmid pMDG-VSV-G were co-transfected into human embryonic kidney 293T cells (HEK293T) to produce the viral particles (Bahi et al., 2004a,b). The viral titres were determined by p24 antigen measurements (KPL, USA). For the in vivo experiments, the different viral stocks were matched for viral particle content and used at $0.2 \mathrm{mg} / \mathrm{mL}$ of $\mathrm{p} 24$, corresponding to $8 \times 10^{9}$ infectious particles/mL.

\section{In vitro assays of $L V-\gamma$ Syn and $L V-D A T$}

The efficiency of the LV- $\gamma$ Syn and LV-DAT were tested in vitro by infection of HEK293T cells: $1 \times 10^{5}$ HEK293T cells were plated per well in six-well plates. The next day, lentiviruses stocks were mixed with $4 \mu \mathrm{g} / \mathrm{mL}$ Polybrene (Sigma, Buchs, Switzerland) with/without $30 \mathrm{ng} / \mathrm{mL}$ doxycycline (Sigma), incubated for $30 \mathrm{~min}$ at room temperature, added to the cells and incubated at $37{ }^{\circ} \mathrm{C}$. After $24 \mathrm{~h}$ the medium was replaced with normal growth medium (with or without $30 \mathrm{ng} / \mathrm{mL}$ doxycycline) and cells were left for a further $48 \mathrm{~h}$. Cells were then collected and used for total RNA isolation (for realtime PCR) or for total protein isolation (for Western blot). Infections with the different lentiviruses were done as follows: (a) doxycycline regulation: cells were co-infected using $0,2,4$ or $8 \mu \mathrm{L}$ of LV- $\gamma$ Syn or LV-DAT stock, and one part of the cells was incubated in the presence of $30 \mathrm{ng} / \mathrm{mL}$ doxycycline and the other part without doxycycline; (b) in vitro silencing of $\gamma$-synuclein: cells were infected with $2 \mu \mathrm{L}$ of LV$\gamma$ Syn stock, either alone or together with $0.5,1,2$ or $4 \mu \mathrm{L}$ of a mix of the three LV- $\gamma$ Syn-siRNAs (mix of LV- $\gamma$ Syn-siRNAs-T1, LV- $\gamma$ SynsiRNAs-T2 and LV- $\gamma$ Syn-siRNAs-T3); (c) in vitro silencing of DAT: cells were infected with $2 \mu \mathrm{L}$ of LV-DAT stock, either alone or together with $0.5,1,2$ or $4 \mu \mathrm{L}$ of a mix of the three LV-DAT-siRNAs (mix of LV-DAT-siRNAs-T1, LV-DAT-siRNAs-T2 and LV-DATsiRNAs-T3).

\section{Quantitative real-time PCR and quantification of $\gamma$-synuclein transcripts}

For quantitative real-time PCR, primer sets for rat $\gamma$-synuclein, DAT, glyceraldehyde-3-phosphate dehydrogenase (GAPDH) or cyclophilin F were designed to amplify 100 - to 200 -bp products, using PRIMER3 software: (http://frodo.wi.mit.edu/cgi-bin/primer3/primer3_http://www. cgi). The following specific primer pairs were used: for rat $\gamma$-synuclein: 5'-CGT CAA CAC AGT GGC CACC-3' and 5'-GGT TCC AAG TCC TCC TTG CG-3'; for rat DAT: $5^{\prime}$-GTT CTA CGG CGT CCA 
GCA-3' and 5'-TGA CCA CGA CCA CAT ACA GG-3'; for rat GAPDH: 5'-ATG ACT CTA CCC ACG GCA AG-3' and 5'-CAT ACT CAG CAC CAG CAT CAC-3'; for rat cyclophilin F: 5'-GTG AGA AGG GCT TTG GCT AC-3' and 5'-TTC TCG TCA GGA AAG CGG-3'. GAPDH and cyclophilin F were used as endogenous controls for normalization. No difference in expression was observed between these two genes in all quantifications.

Total RNA was extracted from the HEK293T cells (for in vitro quantification) and from brains of cocaine-treated animals (for in vivo quantification) using TRIzol Reagent (Invitrogen, Switzerland), including an RNase-free DNase step. RNA was quantified by spectrophotometry, and its integrity verified by agarose gel electrophoresis as visualized with ethidium bromide staining. First strand cDNA was generated from $2 \mu \mathrm{g}$ of total RNA and Oligo(dT $\left.\mathrm{d}_{12-18}\right)$ primer with the M-MLV reverse transcription kit (Invitrogen, Basel, Switzerland) in a total volume of $20 \mu \mathrm{L}$. The reaction product was used for quantitative real-time PCR using the real-time PCR iCycler (Bio-Rad, Reinach, Switzerland). cDNA preparation $(2 \mu \mathrm{L}), 0.5 \mu \mathrm{M}$ of forward and reverse primers, and $10 \mu \mathrm{L}$ of IQ SYBR Green Supermix (Biorad, Reinach, Switzerland) in a total volume of $20 \mu \mathrm{L}$ were applied and PCR was performed as follows: 3 min at $95{ }^{\circ} \mathrm{C}$ (initial denaturation); $20{ }^{\circ} \mathrm{C} / \mathrm{s}$ temperature transition rate up to $95{ }^{\circ} \mathrm{C}$ for $30 \mathrm{~s}, 10 \mathrm{~s}$ at $55{ }^{\circ} \mathrm{C}$ and $30 \mathrm{~s}$ at $56{ }^{\circ} \mathrm{C}$, repeated for 40 cycles (amplification). The PCR reaction was evaluated by melting curve analysis and by checking the PCR products on $2 \%$ agarose gel.

The PCR cycle number at which each assay target reached the threshold detection line was determined ['threshold cycles', $(\mathrm{Ct})$ value]. The $\mathrm{Ct}$ of each candidate was normalized against that of GAPDH or cyclophilin F. To determine the linearity and detection limit of the assay, cDNA samples were amplified for successive 10-fold dilutions in a series of real-time PCRs, using duplicate assay on each dilution, so that the correlation coefficient could be calculated from the standard curve of $\mathrm{Ct}$ values. Comparisons were made between cocaine and saline groups, and significance was calculated using two-tailed Student's $t$-test and the level of statistical significance was set at $P<0.05$. Data were expressed as means $\pm \mathrm{SEM}$. The $\mathrm{Ct}$ for each candidate was calculated as: $\mathrm{Ct}=[\mathrm{Ct}$ (candidate) $-\mathrm{Ct}(\mathrm{GAPDH}$ or cyclophilin F)]. The relative abundance of each target in each protocol can be calculated as the ratio between treated and untreated samples.

\section{Western blotting}

Rat NAc (150-200 mg) or transfected HEK293T cells were homogenized in buffer (in mM: Tris, $50, \mathrm{pH} \mathrm{7.5;} \mathrm{NaCl}, 120 ; \mathrm{CaCl}_{2}, 1.5$; $\mathrm{MgCl}_{2}, 5 ; \mathrm{KCl}, 5$; EDTA, 5) with a protease inhibitor mixture (Sigma, St Louis, MO, USA; $1 \mathrm{~mL} / 20 \mathrm{~g}$ of tissue). Homogenates were solubilized with $1 \%$ digitonin, followed by the addition of secondary solubilization buffer [in mM: Tris, 50, pH 7.6; NaCl, 150; 1\% Nonidet-P40; $0.5 \%$ sodium deoxycholate; EDTA, 2; sodium orthovanadate, 1 ; phenylmethylsulphonyl fluoride (PMSF), 1; 1\% Triton $\mathrm{X}-100]$, and centrifuged at $10000 \mathrm{~g}$ at $4{ }^{\circ} \mathrm{C}$ for $15 \mathrm{~min}$. Solubilized extracts were subjected to sodium dodecyl sulphate-polyacrylamide gel electrophoresis (SDS-PAGE). Blots were blocked with 5\% non-fat dried milk dissolved in TBST (in mM: Tris, $10 ; \mathrm{NaCl}, 150 ; 0.1 \%$ Tween-20) for $1 \mathrm{~h}$ at room temperature, and incubated with primary antibodies for either DAT $(1: 2000$; Abcam, UK) or $\gamma$-synuclein (1: 1000; Abcam) overnight at $4{ }^{\circ} \mathrm{C}$. Blots were washed three times with TBST and incubated for $1 \mathrm{~h}$ with the peroxidase-conjugated secondary antibody solution. Proteins were then visualized using enhanced chemiluminescence (Millipore, Basel, Switzerland). Mem- branes were washed for $30 \mathrm{~min}$ in TBST and placed in stripping solution (glycine-HCl $25 \mathrm{~mm}, \mathrm{pH} 2.0,1 \% \mathrm{SDS}$ ) for $30 \mathrm{~min}$ and used as described above for $\beta$-actin detection ( $1: 4000$; Sigma).

\section{Immunohistochemistry}

For immunohistochemistry assays, rats were decapitated. Brains were quickly removed from the skull, immediately frozen in isopentane (at $-30{ }^{\circ} \mathrm{C}$ for $3 \mathrm{~min}$ ) and kept at $-25{ }^{\circ} \mathrm{C}$. Coronal sections were cut at $14 \mu \mathrm{m}$ in a cryostat and placed on gelatinized glass slides, air-dried at room temperature for $20 \mathrm{~min}$ and kept at $-25{ }^{\circ} \mathrm{C}$ until further processing. Brain sections were fixed for $10 \mathrm{~min}$ in cold acetone and washed three times in $1 \times$ phosphate-buffered saline (PBS). Nonbinding sites were blocked by incubating slices for $1 \mathrm{~h}$ in $1 \times \mathrm{PBS}$ containing $1 \%$ bovine serum albumin, $1 \%$ Triton $\mathrm{X}-100$ and $3 \%$ normal goat serum. Sections were then incubated overnight at $4{ }^{\circ} \mathrm{C}$ with primary antibodies specifically against either $\gamma$-synuclein (1 : 5000; mouse anti- $\gamma$-synuclein antibody, Abcam) or DAT (rabbit anti-DAT antibody, Ab1591P, Chemicon, $1: 5000$ ). Antibodies were diluted in $1 \times$ PBS containing $0.1 \%$ Triton X-100 and $1 \%$ normal goat serum. Thereafter slices were washed three times in $1 \times$ PBS, and incubated for $2 \mathrm{~h}$ in the secondary antibodies (Texas red-conjugated goat anti-rabbit immunoglobulin G, $1: 500$ and FITC-conjugated or Texas-conjugated anti-mouse immunoglobulin G, $1: 500)$ in $1 \times$ PBS containing $0.1 \%$ Triton X-100. The sections were coverslipped with a medium containing glycerol in PBS (AF1 mountant solution, Citifluor). Negative controls included omission or substitution of primary antibodies.

\section{Fluorescence microscopy}

To observe the stained sections, a multifluorescence microscope (Axioplan 2 imaging, Zeiss, Germany) was used with a $40 \times$ objective. Fluorophores used (Texas red and FITC) were detected with the appropriate detecting systems (HAL 100). Texas red was exited by a $570-\mathrm{nm}$ beam and was detected through a light path ranging 600-660 nm. FITC was excited at $495 \mathrm{~nm}$ and was detected through a light path ranging $510-550 \mathrm{~nm}$. Stained sections were visualized, photographed using a multichannel camera (Axiocam, Zeiss, Germany) combined to acquisition software (Axiovision system 3.1) and recorded on CD.

\section{Animal handling and behavioural analysis}

Animals used in this experiment were male Wistar rats weighing 250 280 g. All animal experiments were carried out in accordance with the guidelines and regulations for Animal Experimentation, BAG, Bern, Switzerland. The animals were housed four per cage in clear plastic cages with wire grid lids. Access to food and water was unrestricted. The animals were kept in the animal facility maintained on a $12 \mathrm{~h}$ light : $12 \mathrm{~h}$ dark cycle (lights off at $07.00 \mathrm{~h}$ ). For doxycycline treatment, animals were maintained with $0.02 \%$ doxycycline (Sigma) and 5\% sucrose supplemented in water. During the gene overexpression period, animals were maintained only with $5 \%$ sucrose.

\section{Stereotaxic surgery}

Stereotaxic surgery and injection of the lentiviral vector were performed according to previous publications (Bahi et al., 2004a,b). Briefly, animals were anaesthetized with a mix of ketamine/xylazine 
$(100 \mathrm{mg} / \mathrm{kg} / 10 \mathrm{mg} / \mathrm{kg}$, i.p.), placed in a stereotaxic apparatus (KOP Model 920, Colbourn, USA) and bilaterally injected into the core part of the NAc with $2 \mu \mathrm{L}$ or $4 \mu \mathrm{L}$ of concentrated lentiviral stock $\left(0.2 \mathrm{mg} / \mathrm{mL}\right.$ of $\mathrm{p} 24$, corresponding to $\left.8 \times 10^{9} \mathrm{IU} / \mathrm{mL}\right)$ using a $10-\mu \mathrm{L}$ Hamilton syringe (Hamilton, RN801). The injections were performed bilaterally at the following coordinates, as calculated from bregma and the dura mera: anterior +1.4 ; lateral \pm 1.4 ; ventral -6.8 (Paxinos \& Watson, 1998).

\section{Procedures}

Experiment 1: effects of DAT on cocaine-induced locomotor activity

Four groups of animals $(n=8)$ were prepared: one group was stereotaxically injected with $2 \mu \mathrm{L}$ of LV-GFP (controls); a second group was injected with $2 \mu \mathrm{L}$ of LV-DAT; a third group was injected with $2 \mu \mathrm{L}$ of a mix of all three LV-DAT-siRNAs; and the fourth group was co-injected with $2 \mu \mathrm{L} \mathrm{LV}$-DAT together with $2 \mu \mathrm{L}$ of a mix of the three LV-DAT-siRNAs.

Experiment 2: effects of $\gamma$-synuclein on cocaine-induced locomotor activity

Four groups of animals $(n=8)$ were prepared: one group was stereotaxically injected with $2 \mu \mathrm{L}$ of LV-GFP (controls); a second group was injected with $2 \mu \mathrm{L}$ of $\mathrm{LV}-\gamma \mathrm{Syn}$; a third group was injected with $2 \mu \mathrm{L}$ of a mix of all three LV- $\gamma$ Syn-siRNAs; and the fourth group was injected with $2 \mu \mathrm{L} \mathrm{LV}-\gamma$ Syn together with $2 \mu \mathrm{L}$ of a mix of the three LV- $\gamma$ Syn-siRNAs.

\section{Experiment 3: effects of DAT and $\gamma$-synuclein on cocaine-induced} locomotor activity

Four groups of animals $(n=8)$ were prepared: one group was stereotaxically injected with $2 \mu \mathrm{L}$ of LV-GFP (controls); one group was stereotaxically injected with $2 \mu \mathrm{L}$ of LV-DAT; a third group was injected with $2 \mu \mathrm{L}$ of a mix of all three LV- $\gamma \mathrm{Syn}$; and the fourth group was injected with $2 \mu \mathrm{L}$ LV-DAT together with $2 \mu \mathrm{L}$ of the LV- $\gamma$ Syn.

After a recovering period of 7-12 days, locomotor activity was monitored in daily sessions over $3 \times 5$ days in $43.2 \times 43.2-\mathrm{cm}$ MEDOFA-RS cages (MED Associates, USA) during the dark cycle, according to previously published procedures (Bahi et al., 2004a,b). Animals were fed without doxycycline for 5 days (Session A), then fed doxycycline for 5 days (Session B), and finally fed without doxycycline for 5 days (Session C). Prior to each locomotor activity measurement, the animal was injected with $0.9 \%$ saline solution, placed into the activity-monitoring cage for a $15 \mathrm{~min}$ baseline. After this 15 -min period, the session automatically paused for a few seconds and, during this interval, each subject received cocaine- $\mathrm{HCl}$ (Sigma, $15 \mathrm{mg} / \mathrm{kg}$, i.p.) and was then placed back into the locomotor activity-monitoring cage for a further $60 \mathrm{~min}$. At the end of the third session (Session C), one half of the animals were killed by decapitation. The other half was switched back on doxycycline for $48 \mathrm{~h}$ then killed. During this switch period, animals were still daily administered cocaine and, as for the other group, were killed $24 \mathrm{~h}$ after the last cocaine injection. Brains were used either for immunocytochemistry or qRT-PCR.

\section{In vivo assays of $\gamma$-synuclein}

To assess the efficiency of lentiviruses-mediated expression of $\gamma$-synuclein and DAT in vivo, brains, after death, were either frozen for immunohistochemistry analysis or dissected out and homogenized for protein or qRT-PCR analysis. For molecular analysis, NAc, striatum and ventral tegmental area (VTA) regions were isolated and tissues were used for RNA extraction using TRIzol Reagent (Invitrogen, Basel, Switzerland) or for protein extraction.

\section{Statistical analyses}

Two-way ANOVA was used to compare both lentiviral and drug treatment in the presence or absence of doxycycline, using GraphPad PRISM (V3.0, GraphPad, San Diego, CA, USA). For locomotor activity, two-way ANOVA was used with time as the within-subject factors and lentiviral treatment as the between-subject factors, followed by Bonferroni post hoc tests. For self-administration and real-time PCR analysis, two-way ANOVA were used with drug treatment (water vs doxycycline) as the within-subject factors and lentiviral treatment as the between-subject factors, followed by Bonferroni post hoc tests.

\section{Results}

\section{Cocaine-mediated behavioural changes upon DAT or $\gamma$-synuclein expression}

Lentiviruses used in this study were prepared and tested in vitro for assessing expression or silencing of DAT and $\gamma$-synuclein, in different combinations prior to in vivo assays (see Supplementary material, Appendix S1, Figs S1 and S2, and Table S1).

\section{Experiment 1: effects of DAT on cocaine-induced locomotor activity}

To test the effects of DAT expression changes in the NAc on cocaineinduced locomotor activity, four groups of animals $(n=8)$ were used. A first group of animals, infected with the LV-GFP, served as a control group and expressed the GFP in the NAc in a doxycycline-regulated way. Previous work clearly established that GFP-overexpression in the NAc has no significant effect on locomotor activity, compared with naïve animals (Bahi et al., 2004a,b). One week after surgery, animals were fed $5 \%$ sucrose, chronic cocaine delivery was started and locomotor activity was monitored during five consecutive days. Animals overexpressing GFP displayed a significant induction of locomotor activity upon cocaine injection, with a peak at 4300 counts (Fig. 1 upper panel, Session A. See also supplementary Fig. S3). At the end of this 5 -day session, animals were fed doxycycline $(0.02 \%$ in $5 \%$ sucrose) to switch off ectopic gene expression. For 2 days (switch period), animals received i.p. cocaine injection but locomotor activity was not monitored, in order to achieve a full, doxycycline-mediated switch off of ectopic gene expression. Thereafter, the locomotor activity was monitored for 5 days on animals still fed doxycycline (Session B). Under these conditions, cocaine-mediated locomotor activity from these GFP-overexpressing animals remained unchanged, with a peak at 4700 counts (Fig. 1 upper panel, Session B). After this 5-day session, animals were again submitted to a 2-day switch period during which animals were fed only $5 \%$ sucrose (no doxycycline). Thereafter, locomotor activity was monitored for five consecutive days on animals still fed without doxycycline, and chronically cocaine injected. During this last period (Session C), cocaine-induced locomotor activity also remained unchanged, with a peak at 4800 counts (Fig. 1 upper panel, Session C).

The second group of animals was infected with doxycyclineregulatable LV-DAT in the NAc. In the absence of doxycycline (Session A), overexpression of DAT induced a significant decrease in locomotor activity compared with the control group (LV-GFP-treated group), with a peak at 2600 counts (Fig. 1 upper panels; $P<0.001$, 


\section{Session A (no doxycycline)}

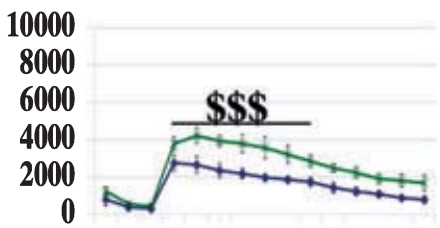

$\begin{array}{llllllll}5 & 15 & 25 & 35 & 45 & 55 & 65 & 75\end{array}$

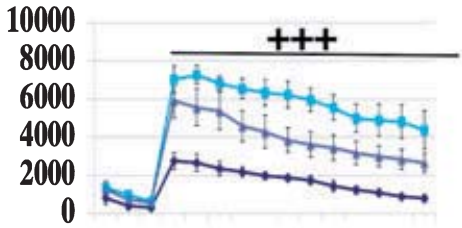

$\begin{array}{llllllll}5 & 15 & 25 & 35 & 45 & 55 & 65 & 75\end{array}$

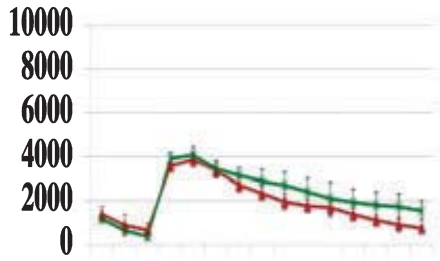

$\begin{array}{llllllll}5 & 15 & 25 & 35 & 45 & 55 & 65 & 75\end{array}$

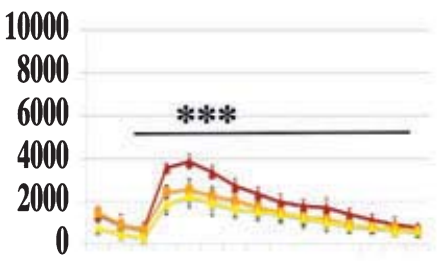

$\begin{array}{llllllll}5 & 15 & 25 & 35 & 45 & 55 & 65 & 75\end{array}$ Time (min)

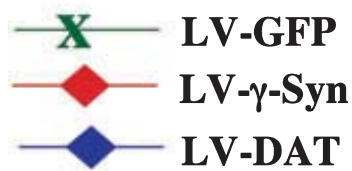

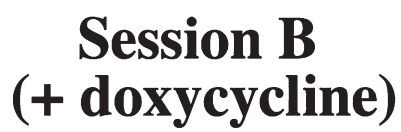
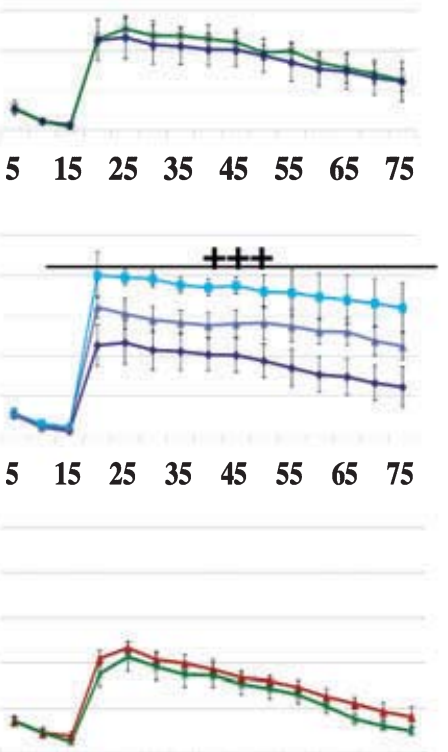

$\begin{array}{llllllll}5 & 15 & 25 & 35 & 45 & 55 & 65 & 75\end{array}$

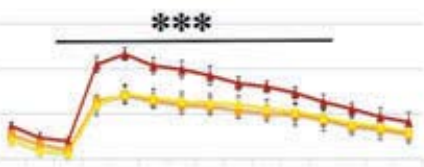

\section{$\begin{array}{llllllll}5 & 15 & 25 & 35 & 45 & 55 & 65 & 75\end{array}$ Time (min)}

\section{LV-DAT + LV-DAT -siRNAs \\ LV- $\gamma$-Syn + LV- $-\gamma$-Syn -siRNAs}

\section{Session C (no doxycycline)}
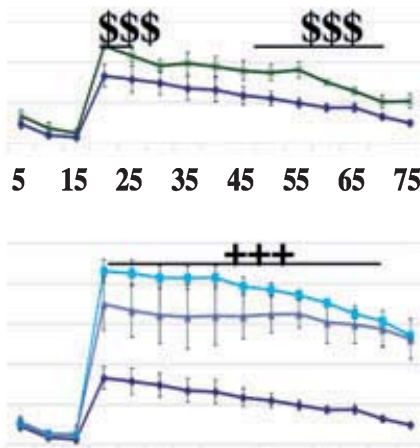

$\begin{array}{llllllll}5 & 15 & 25 & 35 & 45 & 55 & 65 & 75\end{array}$

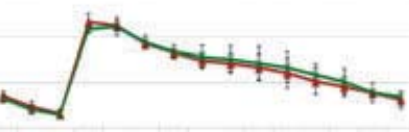

$\begin{array}{llllllll}5 & 15 & 25 & 35 & 45 & 55 & 65 & 75\end{array}$

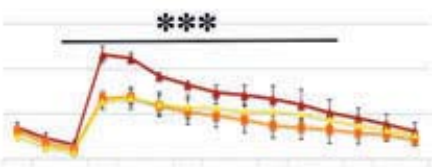

$\begin{array}{llllllll}5 & 15 & 25 & 35 & 45 & 55 & 65 & 75\end{array}$ Time (min)

\section{LV-DAT -siRNAs \\ LV- $\gamma$-Syn -siRNAs}

FIG. 1. In vivo effects of dopamine transporter (DAT) and $\gamma$-synuclein expression in the NAc on cocaine-induced locomotor activity. Values represent means \pm SEM of distance travelled over 5 days of measurement ( $n=8$ animals). One week after surgery, after a recovering period of $7-12$ days, chronic cocaine delivery was started and locomotor activity was monitored in daily sessions over $3 \times 5$ days in $43.2 \times 43.2 \mathrm{~cm}$ MED-OFA-RS cages (MED Associates, USA) during the dark cycle (see Materials and methods). The paradigm comprises an i.p. saline injection prior to the habituation period of 15 min, and an i.p. cocaine injection $(15 \mathrm{mg} / \mathrm{kg}$ ) followed by $1 \mathrm{~h}$ recording. Over 5 days, animals were fed without doxycycline (Session A), yielding to full expression of DAT or $\gamma$-synuclein in the targeted brain area. This was followed by a switch period of 2 days during which animals were fed doxycycline and cocaine injected but not recorded for locomotor activity. During Session B, animals were fed doxycycline in order to switch back ectopic gene expression. At the end of Session B and during two consecutive days animals were submitted again to a switch period during which doxycycline was removed. This was followed by a 5-day period without doxycycline (Session C, regimen identical to Session A). Overexpression of DAT in the NAc (upper panels) induced a significant decrease on cocaine-induced locomotor activity that could be abolished by doxycycline treatment. Silencing of DAT (second row of panels) lead to an increase of cocaine-induced locomotor activity. By contrast, $\gamma$-synuclein overexpression in the NAc (third row of panels) has no consequences in cocaine-induced locomotor activity, whereas silencing of $\gamma$-synuclein (lower panels) lead to a significant decrease in locomotor activity compared with the control group. ${ }^{\$ \$} P<0.001$, as compared with LV-green fluorescent protein (GFP)-injected rats. ${ }^{++} P<0.001$ compared with LV-DAT injected rats. ${ }^{* * *} P<0.001$ compared with LV- $\gamma$ Syn-treated rats. Two-way ANOVA, Bonferonni post hoc tests.

$\left.F_{3,240}=550.8\right)$, whereas under the doxycycline regimen, these same LV-DAT-treated animals demonstrated a locomotor activity very similar to controls (LV-GFP-injected animals) under the same conditions, with a peak at 4700 counts (Fig. 1 upper panels; $P>0.05, F_{3,240}=210.9$ ). After 5 days of the doxycycline regimen, doxycycline was removed, enabling re-expression of lentivirusmediated DAT expression in the NAc, and after a 2-day switch period locomotor activity was monitored for 5 days (Session C): under these conditions the LV-DAT-infected group again demonstrated a significant reduction in locomotor activity, compared with the control group, 
with a peak at 2700 counts as in Session A (Fig. 1 upper panels; $P<0.001, F_{3,240}=828.9$; see also supplementary Fig. S3).

The third group of animals was co-injected with the regulatable LV-DAT together with non-regulatable LV-DAT-siRNAs. In the absence of doxycycline (Session A), a significant increase in locomotor activity was observed, compared with the control group (LV-GFP-treated animals), with a peak at 5900 counts (Fig. 1 second row; $P<0.001$; $F_{3,240}=550.8$ ). Under the doxycycline regimen, only ectopic expression of DAT was turned off, as the LV-DAT-siRNAs are not doxycycline regulatable and thus inhibit endogenous DAT expression; this resulted in a further increase in cocaine-mediated locomotor activity, with a peak at 6400 counts. (Fig. 1 second row; $P<0.001 ; F_{3,240}=210.9$ ). Removal of doxycycline in Session $\mathrm{C}$ induced an increase in locomotor activity, with a peak at 7000 counts (Fig. 1 second row; $P<0.001$; $F_{3,240}=828.9$; see also supplementary Fig. S3).

The fourth group of animals was injected with non-regulatable LV-DAT-siRNAs, in order to knock-down endogenous expression of the DAT in the NAc. In Session A, a dramatic increase in locomotor activity after cocaine injection was observed, as a result of knockdown of endogenous DAT, with a peak at 7100 counts (Fig. 1 second row; $P<0.001 ; F_{3,240}=550.8$ ), which was not affected by doxycycline. In Sessions B and C, peaks at 7700 counts and 8600 counts were observed (Fig. 1 second row; $P<0.001 ; F_{3,240}=210.9$ and $P<0.001 ; F_{3,240}=828.9$, respectively).

\section{Experiment 2: effects of $\gamma$-synuclein on cocaine-induced locomotor activity}

The effects of $\gamma$-synuclein expression on cocaine-induced locomotor activity were tested similarly in four groups of animals $(n=8)$. A first group of animals (control), injected with doxycycline-regulatable LV-GFP in the NAc, demonstrated a cocaine-induced locomotor activity unaffected by doxycycline regimen, with peaks at 4100 counts, 4300 counts and 4400 counts in Sessions A-C, respectively (Fig. 1 third row and supplementary Fig. S3).

The second group, injected with LV- $\gamma$ Syn in the NAc, locally overexpressing $\gamma$-synuclein, displayed locomotor activity similar to the control group during Session A, with a peak at 4700 counts (Fig. 1 third row; $\left.P>0.05 ; F_{4,300}=111.7\right)$. Upon the doxycycline regimen (Session B), leading to switch off of ectopic $\gamma$-synuclein expression, cocaine-induced locomotor activity was not significantly different and very similar to the control group, with a peak at 4700 counts (Fig. 1 third row; $\left.P>0.05 ; F_{4,300}=151.1\right)$. Animals were then switched back to the doxycycline-free regimen (Session $C$ ), leading again to overexpression of $\gamma$-synuclein in the NAc: under these conditions again, locomotor activity was similar to the control group (Fig. 1 third row; $P>0.05 ; F_{4,300}=117.8$; see also supplementary Fig. S3).

The third group of animals was co-injected with a doxycyclineregulatable LV- $\gamma$ Syn together with non-regulatable LV- $\gamma$ Syn-siRNAs. In the absence of doxycycline (Session A), cocaine-induced locomotor activity was strongly reduced and significantly lower than the control group (Fig. 1 last row; $P<0.001 ; F_{4,300}=111.7$; see also supplementary Fig. S3). Switch to the doxycycline regimen (Session B) switches off ectopic $\gamma$-synuclein overexpression, maintaining silencing of endogenous $\gamma$-synuclein; under these conditions, rats still demonstrated a significant decrease in locomotor activity, compared with GFP-infected animals (Fig. 1 last row; $P<0.001 ; F_{4,300}=151.1$ ), which remained unaffected upon removal of doxycycline (Session $\mathrm{C}$; Fig. 1 last row; $P>0.05 ; F_{4,300}=117.8$ ).

A fourth group of animals was injected only with non-regulatable LV- $\gamma$ Syn-siRNAs, in order to knock-down the expression of endogenous $\gamma$-synuclein in the NAc. During Session A, animals displayed a significant reduction of cocaine-induced locomotor activity, compared with the control group, with a peak at 2200 counts (Fig. 1 last row; $\left.P<0.001 ; F_{4,300}=111.7\right)$. Despite the fact that these lentiviruses were not doxycycline regulatable, animals were nevertheless submitted to the doxycycline regimen, for effective comparison between groups. Doxycycline switch did not significantly affect locomotor activity and, during Session B, these rats still showed a significant decrease in locomotor activity, as compared with LV-GFP-treated animals (Fig. 1 last row; $P<0.001 ; F_{4,300}=151.1$ ). This was unmodified upon removal of doxycycline (Session C; Fig. 1 last row; $P>0.05 ; F_{4,300}=117.8$; see also supplementary Fig. S3). These results demonstrated that local changes in $\gamma$-synuclein expression may affect cocaine-induced locomotor activity. In order to establish whether these changes would synergize or antagonize those observed upon DAT manipulation, the impact of co-injection of LV-DAT and LV- $\gamma$ Syn in the NAc on cocaine-induced locomotor activity was investigated.

\section{Experiment 3: effects of DAT and $\gamma$-synuclein on cocaine-induced locomotor activity}

To elucidate putative interactions between DAT and $\gamma$-synuclein and their consequences on behaviour, four groups of animals $(n=8)$ were used. The control group, injected with LV-GFP in the NAc, displayed doxycycline-independent cocaine-induced locomotor activity, with a peak at 4400 counts during all three sessions (Fig. 2 upper and middle rows).

The second group of animals was injected with LV-DAT in the NAc, leading to doxycycline-regulatable overexpression of DAT in this brain area. Animals fed only 5\% sucrose (Session A) displayed a significant decrease in cocaine-induced locomotor activity, with a peak at 2900 counts (Fig. 2 upper row; $P<0.001 ; F_{3,240}=227.1$ ). The doxycycline regimen, that switches off ectopic DAT expression, induced normal locomotor activity, comparable to that recorded in the control group (Fig. 2, upper row; $P>0.05 ; F_{3,240}=92.7$ ). Removal of doxycycline for these same animals, thus re-instating DAT overexpression in the NAc, restored the initial significant decrease in locomotor activity, as compared with the control group, with a peak at 3000 counts (Fig. 2, upper row; $P<0.01 ; F_{3,240}=441.1$; see also supplementary Fig. S2). These results were totally similar to those obtained in the previous experiments (Fig. 1), confirming the strong impact (hypolocomotion) of DAT overexpression on cocaine-induced locomotor activity.

The third group was injected with the LV- $\gamma$ Syn in the NAc, submitted to the same regimen as described above. As discussed previously (Fig. 1), the induction of ectopic expression of $\gamma$-synuclein in this area had apparently no consequences on cocaine-induced locomotor activity (Sessions A-C), as no differences were detected on LV- $\gamma$ Syn-treated animals compared with LV-GFP-injected rats (Fig. 2; middle rows, $P>0.05$ ).

The fourth group of animals was co-injected with LV-DAT and LV- $\gamma$ Syn in the NAc. Surprisingly, locomotor activity recorded during Session A was strongly increased, compared with the control group, with a peak at 5900 counts (Fig. 2, lower rows; $P>0.001$; $\left.F_{3,240}=277.1\right)$. Thus, DAT, which reduces locomotion, enables $\gamma$-synuclein, which has no effect alone, to increase locomotion. Despite its oddity, these data could be fully reproduced in another laboratory (data not shown). Doxycycline treatment (Session B) totally abolished the observed hyperlocomotion, and the locomotor activity returned to a basal level comparable to that observed in the LV-GFP group, with a peak at 5300 counts (Fig. 2, lower rows; $P>0.05$; $F_{3,240}=92.7$ ). Removal of doxycycline (Session C) led to an 


\section{Session A (no doxycycline)}

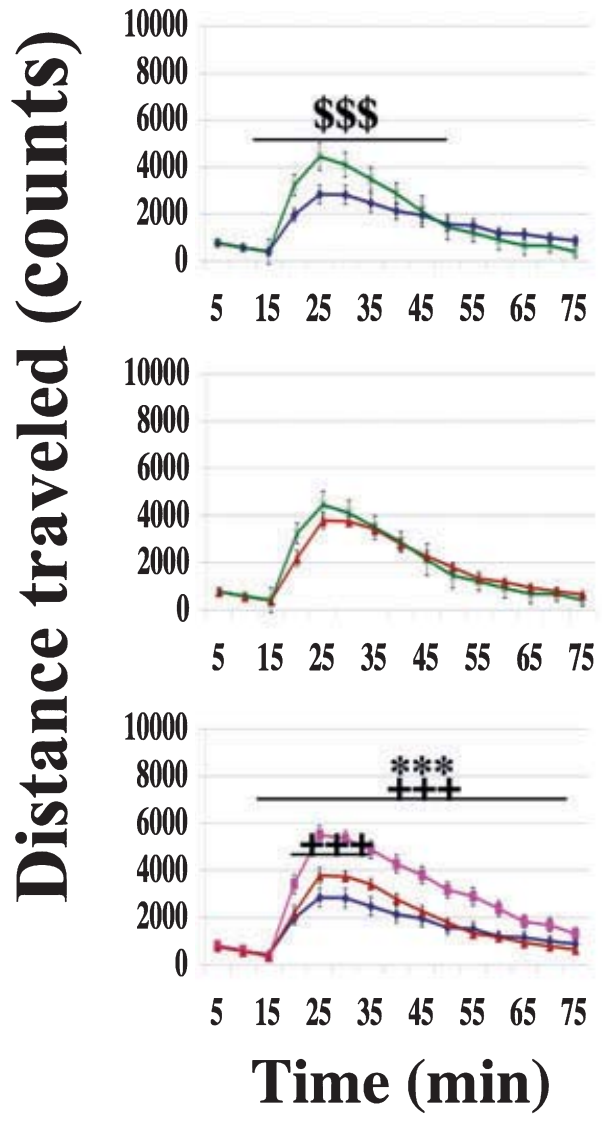

\section{Session B (+ doxycycline)}

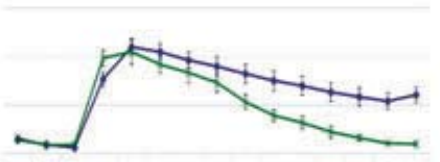

$\begin{array}{llllllll}5 & 15 & 25 & 35 & 45 & 55 & 65 & 75\end{array}$

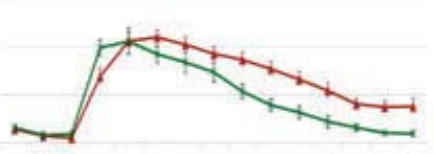

$\begin{array}{llllllll}5 & 15 & 25 & 35 & 45 & 55 & 65 & 75\end{array}$

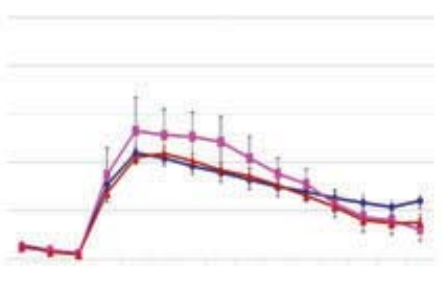

$\begin{array}{llllllll}5 & 15 & 25 & 35 & 45 & 55 & 65 & 75\end{array}$ Time (min)

\section{Session C (no doxycycline)}

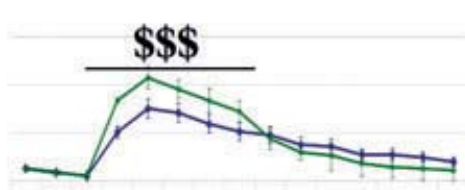

$\begin{array}{llllllll}5 & 15 & 25 & 35 & 45 & 55 & 65 & 75\end{array}$

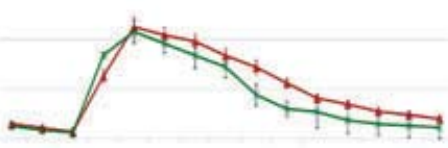

$\begin{array}{llllllll}5 & 15 & 25 & 35 & 45 & 55 & 65 & 75\end{array}$

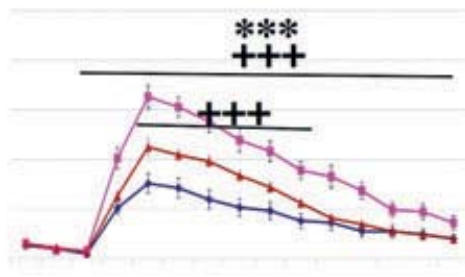

$\begin{array}{llllllll}5 & 15 & 25 & 35 & 45 & 55 & 65 & 75\end{array}$ Time (min)
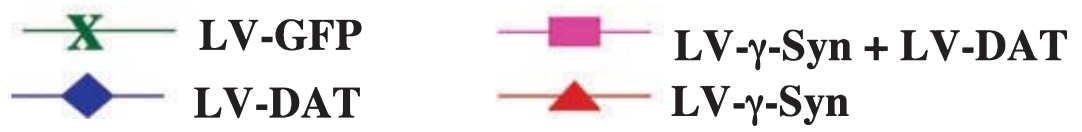

FIG. 2. In vivo effects of dopamine transporter (DAT) and $\gamma$-synuclein overexpression in the NAc on cocaine-induced locomotor activity. Values represent means \pm SEM distance travelled over 5 days of measurement $(n=8$ animals). Four groups of animals were injected bilaterally in the NAc with either LV-DAT alone, LV- $\gamma$ Syn alone, LV-GFP alone or LV-DAT together with LV- $\gamma$ Syn. One week after surgery, cocaine-induced locomotor activity was measured under a regimen identical to Fig. 1. During Sessions A and C animals were fed without doxycycline yielding to full expression of DAT and $\gamma$-synuclein, whereas during Session B animals were fed with doxycycline and ectopic genes expression is suppressed. Overexpression of DAT in the NAc induced a decrease in cocaine-induced locomotor activity (upper panels), whereas overexpression of $\gamma$-synuclein alone in the same brain area has no consequences on locomotor activity (middle panels). Note that coexpression of DAT and $\gamma$-synuclein in the NAc (lower panels) leads to a significant increase in cocaine-induced locomotor activity; these changes could be totally abolished by doxycycline treatment. ${ }^{\$ \$ \$} P<0.001$ compared with LV-green fluorescent protein (GFP)-injected rats. ${ }^{+++} P<0.001$ compared with LV-DAT-injected rats; ${ }^{* * *} P<0.001$ compared with LV- $\gamma$ Syn-treated rats. Two-way ANOVA, Bonferonni post hoc tests.

overexpression of DAT and $\gamma$-synuclein proteins that reinstated hyperlocomotor activity back to its initial levels, with a peak at 6500 counts after cocaine injection (Fig. 2, lower rows; $P<0.001$; $F_{3,240}=92.7$; see also supplementary Fig. S3). These data clearly suggest that this cocaine-induced hyperlocomotion, mediated by overexpression of both DAT and $\gamma$-synuclein together, could be caused by (direct or indirect) interaction.

\section{mRNA expression in vivo}

\section{DAT mRNA expression in Experiment 1}

At the end of behavioural analyses, half of the animals from each groups were killed, the second half of the animals was fed doxycycline for $48 \mathrm{~h}$ (turning off lentivirus-mediated gene overexpression in the NAc), then killed. After killing, brains were removed, NAc tissues were dissected out and used for total RNA extraction, Western blots analysis or immunohistochemistry analysis After reverse transcription, mRNA expression levels were measured by means of qRT-PCR. Data are shown in Fig. 3A-D.

When animals had been injected with LV-GFP, DAT mRNA expression was detected with a ratio of 1.1. Animals from this group that were switched to doxycycline displayed the same ratio of DAT expression. The ratios of cyclophilin $\mathrm{F}$ and GAPDH mRNA remained unchanged over cocaine-treated control animals in both cases (not shown).

When animals had been injected with LV-DAT alone, strong induction of DAT mRNA expression was observed in the absence of 


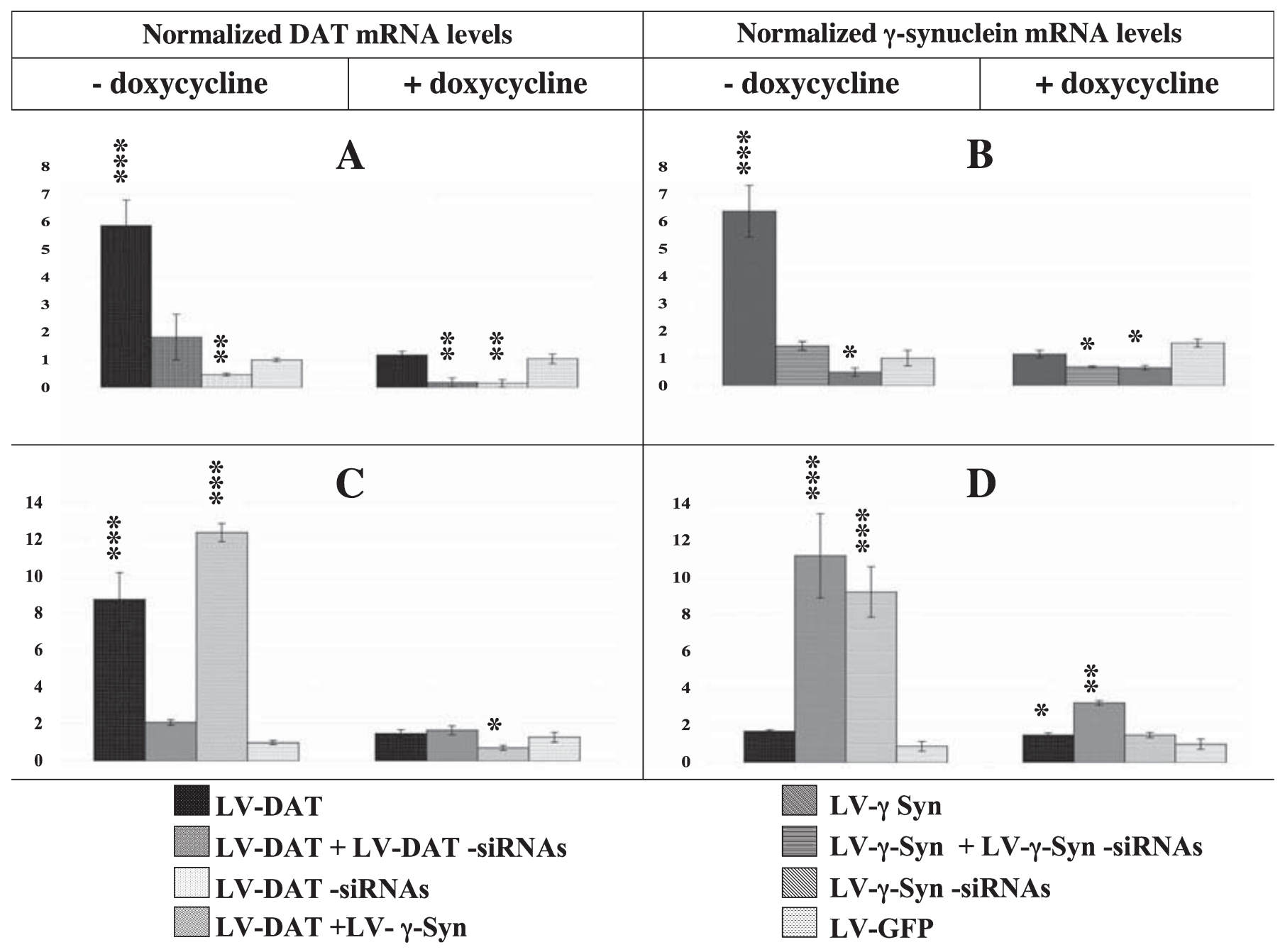

FIG. 3. mRNA quantification of in vivo gene expression from NAc-infected animals. Quantifications by means of qRT-PCR. At the end of the behavioural tests, animals were killed and NAc was dissected out. Total RNA was extracted using the Trizol method, cDNA was prepared and transcripts levels were quantified using the real-time PCR method with specific primers. Quantitative mRNA levels were normalized against cyclophilin F. GAPDH mRNA was also analysed as an endogenous control. Data represent means \pm SEM. (A) In vivo expression of dopamine transporter (DAT) mRNA from NAc of LV-DAT- and/or LV-DAT-siRNAsinfected rats. ${ }^{* * *} P<0.001,{ }^{* * P}>0.01$ compared with $\mathrm{LV}$-green fluorescent protein (GFP)-injected rats. (B) In vivo expression of $\gamma$-synuclein mRNA from NAc of LV- $\gamma$ Syn- and/or LV-siRNAs- $\gamma$ Syn-infected rats. $* * * P<0.001, * P<0.05$ compared with LV-GFP-injected rats. (C) In vivo expression of DAT mRNA from NAc of rats injected with LV- $\gamma$ Syn, LV-DAT, LV-DAT + LV- $\gamma$ Syn or LV-GFP. $* * * P<0.001, * * P<0.01, * P<0.05$ compared with LV-GFP-injected rats. (D) In vivo expression of $\gamma$-synuclein mRNA from NAc of rats injected with LV- $\gamma$ Syn, LV-DAT, LV-DAT + LV- $\gamma$ Syn or LV-GFP. ${ }^{* * *} P<0.001,{ }^{* *} P<0.01$, $* P<0.05$ compared with LV-GFP-injected rats.

doxycycline, with a ratio of $\sim 5.9$ compared with LV-GFP-treated controls (Fig. 3A; $P<0.001 ; F_{7,48}=26.9$ ). This expression level dropped to 1.2-fold upon the doxycycline regimen, equivalent to the ratio observed for GFP-treated animals. GAPDH mRNA expression was not affected, and ratios of 1 and 0.9 (in the absence or presence of doxycycline, respectively) were found, similar to those observed in the previous group (not shown).

When animals had been injected with LV-DAT together with a mix of LV-DAT-siRNAs a decrease of DAT expression was found in the absence of doxycycline, resulting in a ratio of $\sim 1.8 \quad(P<0.01$; $\left.F_{7,48}=26.9\right)$. With doxycycline treatment, complete inhibition of DAT was found, with ratios $\sim 0.2$ (Fig. $3 \mathrm{~A} ; P<0.01 ; F_{7,48}=26.9$ ). Indeed, doxycycline blocks exogenous, lentivirus-mediated DAT overexpression - but not endogenously expressed DAT - while silencers also block endogenous DAT. Under these conditions, the other targets (GAPDH, etc.) were not modified, indicating that LV-DAT-siRNAs had no effects on their expression levels.
Finally, when animals have been injected with LV-DAT-siRNAs only (without LV-DAT), the DAT mRNA expression level dropped to $\sim 0.5$ and even to $\sim 0.15$ with doxycycline (Fig. $3 \mathrm{~A} ; P<0.01$ ), corresponding to full silencing of endogenous DAT mRNA expression, whereas GAPDH mRNA was not modified.

\section{Synuclein $m R N A$ expression in Experiment 2}

Animals injected with LV-GFP displayed $\gamma$-synuclein mRNA expression with a ratio of 1.04 in the absence of doxycycline. When switched to doxycycline the same ratio (1.1) of $\gamma$-synuclein mRNA expression was found. No changes in GAPDH ratios were found in this group in both cases (Fig. 3B).

Animals injected with LV- $\gamma$ Syn displayed a strong increase of $\gamma$-synuclein mRNA expression, with a ratio of 6.9 in the absence of doxycycline, whereas in the presence of doxycycline this ratio dropped to 1.2 (Fig. 3B; $P<0.001 ; F_{7,48}=8.9$ ). Thus, the lack of 
behavioural changes in LV- $\gamma$ Syn-injected rats was not due to a trouble of LV- $\gamma$ Syn expression.

Animals co-injected with LV- $\gamma$ Syn and LV- $\gamma$ Syn-siRNAs displayed expression levels of $\gamma$-synuclein transcripts similar to those found in control groups, with a ratio of 1.5 in the absence of doxycycline (Fig. 3B; $P>0.05 ; F_{7,48}=8.9$ ). Under the doxycycline regimen, a significant decrease in $\gamma$-synuclein transcripts level was observed, with a ratio of $\sim 0.6$ (Fig. 3B; $P<0.05 ; F_{7,48}=8.9$ ).

Animals injected with LV- $\gamma$ Syn-siRNAs alone displayed an inhibition of $\gamma$-synuclein expression with ratios of 0.5 and 0.6 in the presence or absence of doxycycline, respectively (Fig. 3B; $P<0.05$; $F_{7,48}=8.9$ ). In all these groups no changes in GAPDH transcripts levels were observed.

\section{$m R N A$ expression in Experiment 3}

In this experiment, in LV-GFP-injected animals, DAT expression was not affected by doxycycline regimen and showed a stable ratio of $\sim 0.9$ under all conditions (Fig. 3C). Animals injected with LV-DAT in the NAc displayed a high increase of DAT mRNA expression in doxycycline-free regimen, with a ratio of 9.4 (Fig. 3C; $P<0.001$; $F_{7,48}=105.5$ ), which returned to a basal level comparable to that observed in the control group in the presence of doxycycline, with a ratio of 1.3 (Fig. $3 C ; P>0.05 ; F_{7,48}=105.5$ ). Animals co-injected with LV-DAT and LV- $\gamma$ Syn displayed higher levels of DAT expression in the absence of doxycycline, with a ratio of 11.1 (Fig. 3C; $\left.P<0.001 ; F_{7,48}=105.5\right)$ and a ratio of 0.6 in the presence of doxycycline, significantly lower than that observed in the control group (Fig. $3 \mathrm{C} ; P<0.05 ; F_{7,48}=105.5$ ).

Under these conditions, $\gamma$-synuclein mRNA expression was significantly increased in animals injected with LV- $\gamma$ Syn (Fig. 3B) or in the group of animals co-injected with LV-DAT and LV- $\gamma$ Syn (Fig. 3D), with ratios of 11.2 and 9.2, respectively, in the absence of doxycycline $\left(P<0.001 ; F_{7,48}=105.5\right)$, and a ratio of 1.5 in the co-injected group under the doxycycline regimen (Fig. 3D; $P>0.05 ; F_{7,48}=105.5$ ), similar to that observed in the control group. Interestingly, however, under the doxycycline regimen the LV- $\gamma$ Syn group displayed a ratio of 3.2, still significantly higher than that observed for the control LV-GFP group (Fig. 3D; $P<0.05 ; F_{7,48}=105.5$ ).

\section{Protein expression in vivo}

Protein expression was assessed after behavioural analysis on animals from all groups described above. After death, brains were dissected out, and the NAcs were used for Western blot analysis.

LV- $\gamma$ Syn injection in the NAc resulted in a significant increase of $\gamma$-synuclein protein expression in that region, compared with LV-GFPinjected rats (Fig. 4A and C; $P<0.001 ; F_{5,24}=37.4$ ). Under the doxycycline regimen, this increase was abolished and $\gamma$-synuclein protein levels returned back to basal levels observed in the control group (Fig. 4B; $P>0.05 ; F_{5,24}=37.4$ ). Furthermore, injection of LV$\gamma$ Syn-siRNAs in the NAc resulted in drastic $\gamma$-synuclein protein knock-down under all regimens, with ratios significantly lower than those observed for LV-GFP-injected rats (Fig. 4A-C; $P<0.01$; $\left.F_{5,24}=37.4\right)$. Co-injection of LV- $\gamma$ Syn and LV- $\gamma$ Syn-siRNAs produced no significant differences in $\gamma$-synuclein protein levels under all regimens, as compared with the control, LV-GFP-injected group (Fig. 4A-C; $P>0.05 ; F_{5,24}=37.4$ ).

When rats were injected with LV-DAT in the NAc, a significant increase of DAT protein levels was found in that region in the absence of doxycycline [Fig. 4A (right panel) and D; $P<0.001$; $\left.F_{5,24}=26.8\right]$. In the presence of doxycycline, DAT expression was strongly reduced, and protein levels were similar to those observed in the control (LV-GFP-treated) group [Fig. 4B (right panel) and D; $\left.P>0.05 ; F_{5,24}=26.8\right]$. Animals injected with LV-DAT-siRNAs displayed a strong decrease of DAT protein expression under all doxycycline regimens, as compared with the control group (Fig. 4A, B and $\mathrm{D} ; P<0.001 ; F_{5,24}=26.8$ ). Interestingly, no significant effects on DAT protein expression were found when LV-DAT has been co-injected with LV-DAT-siRNAs (Fig. 4D).

Co-injection of LV-DAT and LV- $\gamma$ Syn in the NAc led to strong induction of expression of both proteins in that region. In this experiment, animals displayed DAT and $\gamma$-synuclein protein levels significantly different to LV-GFP-injected rats (Fig. 4A; $P<0.001$ ). Doxycycline treatment of rats from this co-injected group resulted in a decrease of $\gamma$-synuclein protein expression that returned to basal levels, whereas the expression of DAT protein showed a weaker decrease in expression but its level was still significantly different compared with the control group (Fig. 4A and E; $P<0.05$ ).

\section{Immunohistochemistry analysis}

Immunohistochemistry was performed on brains from animals treated with LV- $\gamma$ Syn together with LV-DAT (Fig. 5). Animals treated with LV- $\gamma$ Syn and LV-DAT display a very strong overexpression of both proteins in the NAc. Low-magnification immunolabelled pictures demonstrate full infection of the entire NAc by both lentiviruses (Fig. 5A). In the NAc the expression of $\gamma$-synuclein (Fig. 5A red, left) and DAT (Fig. 5A brown, right) covers the entire zone of interest, but expression is limited in that zone, demonstrating again the low capacity of diffusion of lentiviruses within the zone of injection. Clearly lentiviruses do not diffuse into other parts of the brain away from the zone of injection. This enables to drive the regulatable expression of the genes of interest ( $\gamma$-synuclein and DAT) without any spreading into other regions, which might compromise interpretation of observed behavioural changes. High-magnification views of gene expression in the NAc show that overexpression of $\gamma$-synuclein and DAT is almost exclusively neuronal within the NAc, and demonstrate the high expression of $\gamma$-synuclein (red, left) and DAT (green, middle) proteins and their co-localization within neurites (Fig. 5B, upper panels) as well as at the cell bodies (Fig. 5B, lower panels). The subcellular co-localization of $\gamma$-synuclein and DAT protein expression could explain the synergic effects found in the locomotor activity analysis.

\section{Expression in the VTA}

In this study lentiviruses have been injected in the NAc and changes in gene expression are observed in this brain region (Figs 3-5). Lentivirus infection is very limited to the targeted brain area (Fig. 5). However, retrotransport of virus from infected accumbal dopaminergic neurons back to the VTA is observed, and ectopic, doxycycline-regulatable, $\gamma$-synuclein and DAT gene expression (or silencing) in the VTA is found in the different groups, as shown by immunocytochemistry and Western blot analysis from tissue of that region (supplementary Fig. S4).

\section{Discussion}

Our results demonstrate that local changes in $\gamma$-synuclein expression may affect cocaine-induced locomotor activity. Overexpression of $\gamma$ synuclein in the NAc alone had no effect, while local silencing lead to a significant decrease in cocaine-induced locomotor activity $(47 \%$ 


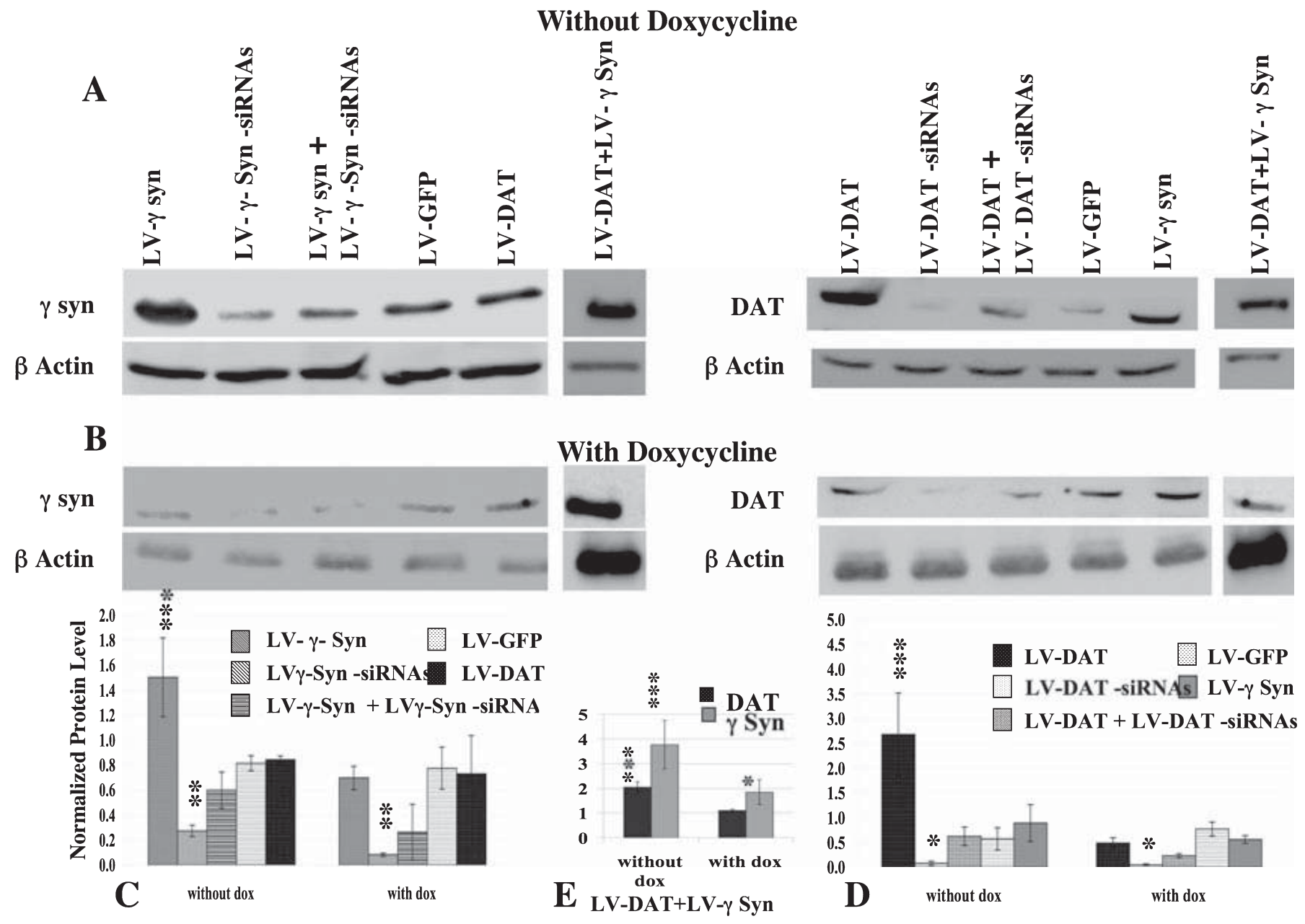

FIG. 4. Western blot analysis of dopamine transporter (DAT) and $\gamma$-synuclein protein expression in the NAc of infected animals. Western blots from gels loaded with $20 \mu \mathrm{g}$ protein/lane were analysed for either DAT or $\gamma$-synuclein expression, and reprobed with $\beta$-actin antibody to compare DAT or $\gamma$-synuclein expression in lentivirus-infected NAc of rats. (A) Immunoblots of DAT and $\gamma$-synuclein expression from NAc of animals in doxycycline-free conditions. (B) Immunoblots of DAT and $\gamma$-synuclein expression from NAc of animals under doxycycline treatment. $\gamma$-Synuclein and DAT signals were estimated using multianalyst software and normalized against the $\beta$-actin signal (see Materials and methods for details). Data represent means \pm SEM. (C) In vivo $\gamma$-synuclein protein levels quantification. ${ }^{* * *} P<0.001,{ }^{* *} P<0.01$ : semiquantitative $\gamma$-synuclein protein quantification vs $\gamma$-synuclein quantification detected from LV-green fluorescent protein (GFP)injected rats. (D) In vivo DAT protein levels quantification. ${ }^{* *} P<0.001,{ }^{*} P<0.01$ : semiquantitative DAT protein quantification vs DAT quantification detected from LV-GFP-injected rats. (E) In vivo $\gamma$-synuclein and DAT protein levels quantification in the group co-infected with LV-DAT together with LV- $\gamma$ Syn. $*_{* *} P<0.001, * * P<0.05$, semiquantitative $\gamma$-synuclein or DAT protein quantification vs $\gamma$-synuclein or DAT quantification detected from LV-GFP-injected rats.

decrease compared with controls). Surprisingly, co-injection of lentiviruses expressing DAT and $\gamma$-synuclein - leading to overexpression of both proteins in the NAc - resulted in a strong increase in cocaine-induced rat locomotor activity ( $52 \%$ increase compared with controls). This increase in locomotor activity was abolished upon locally silencing these genes. Thus, $\gamma$-synuclein, which by itself has no effect alone, enables DAT, which reduces locomotion, to increase locomotion; despite its oddity, these data could be fully reproduced (including qRT-PCR and Western blot analysis) in another laboratory under the same paradigms and also under different paradigms, e.g. self-administration (collaboration with Dr V. Deroche-Gamonet, CRI U862, Bordeaux, unpublished). This suggests a synergistic role of both proteins, possibly mediated through a direct interaction. By contrast previous works have shown that overexpression of $\alpha$-synuclein in the NAc induces a $45 \%$ increase in locomotor activity, while local silencing of $\alpha$-synuclein in the NAc resulted in significant hypolocomotion activity and a decrease in self-administration (Boyer \& Dreyer, 2007).
Together these data demonstrate that both $\alpha$ - and $\gamma$-synuclein modulate cocaine-induced behavioural effects, in different ways but probably by interaction with DAT (Lavedan, 1998; George, 2001). It has been well established that synuclein activity helps regulate the dopaminergic system (Brenz-Verca et al., 2003; Ferguson, 2003; Wersinger \& Sidhu, 2003; Wersinger et al., 2003a, b). $\alpha$-Synuclein, serving as a chaperone protein, is involved in the regulation of the activity of DAT and of tyrosine hydroxylase, which controls the level of monoamine neurotransmitters in the brain (Perez et al., 2002; Liang et al., 2003; Mash et al., 2003). Qin et al. (2005) demonstrated concomitant regulation of $\alpha$-synuclein and DAT binding and function in human striatal synaptic terminals isolated from cocaine abusers, indicating that overexpression of $\alpha$-synuclein may play a role in cocaine-induced plasticity and regulation of dopamine synaptic tone. The DAT represents the major presynaptic component involved in terminating dopaminergic neurotransmission through facilitating the reuptake of synaptic dopamine (Horn, 1990; Chen \& Reith, 2000), and thus may bolster the production of reactive free radical 

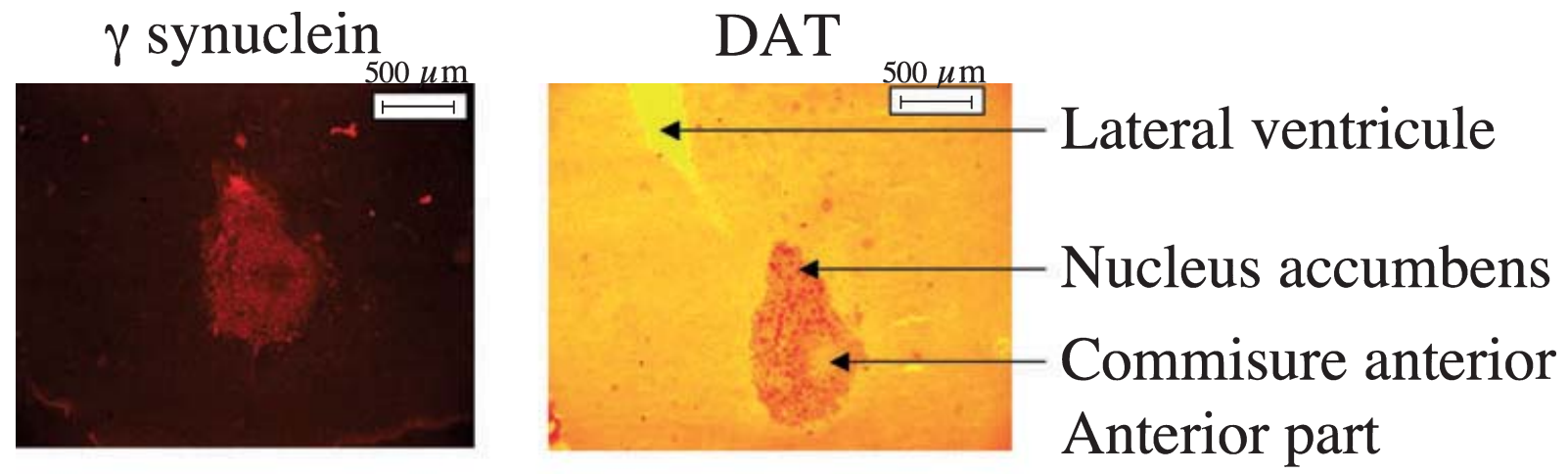

B
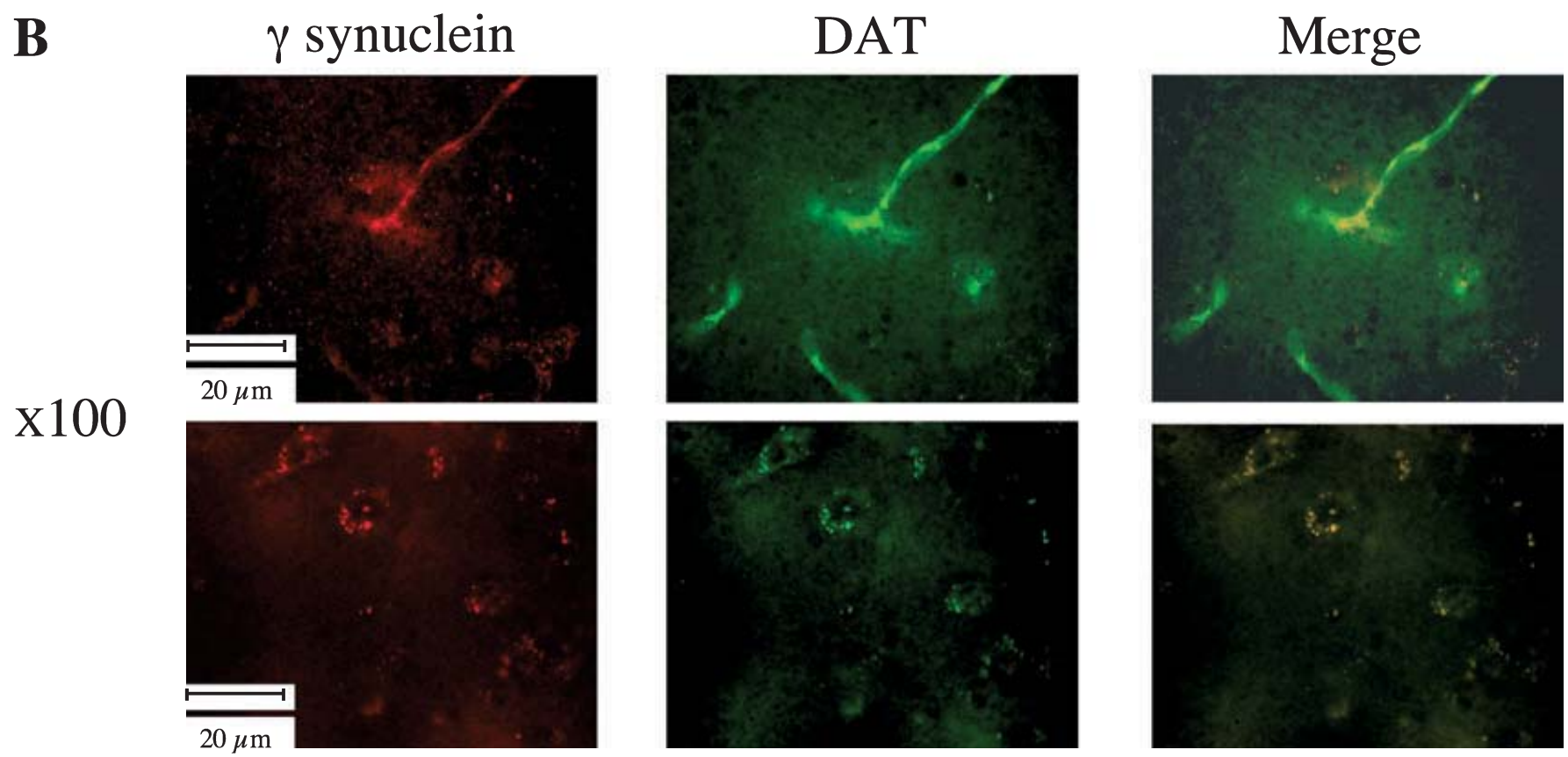

FIG. 5. Immunohistochemistry of $\gamma$-synuclein and dopamine transporter (DAT) expression in the NAc. Immunohistochemistry was performed after in vivo delivery of LV- $\gamma$ Syn $(2 \mu \mathrm{L})$ combined with LV-DAT $(2 \mu \mathrm{L})$ Lentiviruses were injected into the NAc according to the coordinates described in the Materials and methods. Animals used for behavioural analysis were fed either $5 \%$ sucrose or $5 \%$ sucrose $/ 0.02 \%$ doxycycline (see text for details). Animals were killed at the end of behavioural analyses; brains were dissected out and processed for immunohistochemistry (see Materials and methods). (A) Low-magnification expression of $\gamma$-synuclein (left) and DAT (right) in the NAc. Sections were double-labelled using $\gamma$-synuclein (red) or DAT antibody (brown), and revealed by immunofluorescence (Texas-red for $\gamma \mathrm{Syn}$ ) and HRP-DAB (brown, for DAT). Pictures were taken at $2.5 \times$ magnitude. (B) High-magnification expression of $\gamma$-synuclein (left) and DAT (middle) and merge (right) shows co-localization of $\gamma$-synuclein and DAT expression. Brain sections from animals fed 5\% sucrose were labelled using antibodies against $\gamma$-synuclein (red) and DAT (green), and were visualized by fluorescence microscopy at $100 \times$ magnitude. Lentiviral overexpression of $\gamma$-synuclein and DAT shows an increase in $\gamma$-synuclein expression (red) that is co-localized with the DAT labelling (green). The first row demonstrates the co-localization (merge, yellow) of both proteins in neurites, whereas the second row shows the co-localization (merge, yellow) at the plasma membrane of the cell body.

metabolites that would induce neurotoxicity of dopaminergic cells. Therefore, regulation of DAT function plays a critical role in the aetiology of dopamine-related neurological diseases (such as Parkinson's disease).

DAT is expressed at presynaptic dopaminergic terminals in the NAc and produced by dopaminergic neurons projecting from the VTA. In addition, very low levels of DAT mRNA in the NAc have been described in naïve animals (Maggos et al., 1998), in agreement with our observations. Under our experimental conditions, however, we cannot rule out the possibility that Glu- and $\gamma$-aminobutyric acid (GABA)-ergic neurons also take up the virus and that these neurons artificially express DAT in the NAc, whereas under normal conditions DAT would only be expressed by dopaminergic neurons in this brain area. Nevertheless, this does not alter our observations that overex- pression of DAT in the NAc, by modifying synaptic levels of dopamine, affects cocaine-induced behaviour. Our data indicate that locally silencing $\alpha$ - or $\gamma$-synuclein induces distinct behavioural changes in cocaine-mediated locomotor activity. In contrast to $\alpha$-synuclein, $\gamma$-synuclein (or parkin) is an E3 ligase that mediates the ubiquitination of proteins, targeting them for degradation, and plays a protective role against dopamine-induced oxidative stress and apoptosis and against $\alpha$-synuclein toxicity (Petrucelli et al., 2002; Oluwatosin-Chigbu et al., 2003; Jiang et al., 2004; LoBianco et al., 2002; Yamada et al., 2005). In addition, $\gamma$-synuclein can affect $\alpha$-synuclein-mediated toxicity (Choi et al., 2001; Chung et al., 2001; Shimura et al., 2001; Petrucelli et al., 2002; Kim et al., 2002), suggesting functional cross-talk between $\gamma$-synuclein and $\alpha$-synuclein. In cocaine paradigms, induction of both $\alpha$ - and $\gamma$-synuclein has been 
observed (Brenz-Verca et al., 2003); however, to our knowledge fibrillation of synucleins in cocaine-treated animals has not been observed. From this, one may imply that cocaine induces $\alpha$-synuclein for promoting DAT-trafficking, but this is balanced by concomitant $\gamma$-synuclein expression.

$\gamma$-Synuclein mediates both classical K48 ubiquitination of substrate proteins (that leads to proteasomal degradation), and also non-classical K63 polyubiquitination (Lim et al., 2005), which diverts proteins away from proteasomal degradation and may have roles in DNA repair, translational regulation and endocytosis (Pickart, 2000). Ubiquitination may play a role in protein trafficking (Aguilar \& Wendland, 2003; Hicke \& Dunn, 2003) and in targeting proteins to endosomes from the plasma membrane (Hicke, 2001). As a matter of fact, a major mechanism in regulating DAT function is through DAT trafficking. The primary mechanism whereby the uptake velocity of the presynaptic DAT is decreased or increased is via the rapid internalization of cell surface DAT into various intracellular compartments or mobilization of DAT to the cell surface from these pools (Pristupa et al., 1998; Daniels \& Amara, 1999; Melikian \& Buckley, 1999). The $\alpha$-synuclein/DAT complex decreases $K_{\mathrm{m}}$ of DAT for dopamine and facilitates the plasma membrane clustering of the DAT, thereby accelerating cellular dopamine uptake (Wersinger \& Sidhu, 2003). Enhancement of $\left[{ }^{3} \mathrm{H}\right]$ dopamine uptake by $\alpha$-synuclein has been described, as a consequence of increased DAT found at the plasma membrane (George, 2001; Pinhasov et al., 2005). We have shown that an increase in $\alpha$-synuclein expression strongly correlates with behavioural changes observed under different drug administration paradigms (Boyer \& Dreyer, 2007). Local overexpression of $\alpha$-synuclein in the NAc rapidly induces increased locomotor response to cocaine and increased self-administration of the drug, whereas selective knockdown of $\alpha$-synuclein in the NAc strongly affects behaviour (Boyer $\&$ Dreyer, 2007). It has been shown that $\gamma$-synuclein blocks the $\alpha$-synuclein-induced enhancement of DAT uptake by decreasing the DAT membrane expression (Moszczynska et al., 2007). This is mediated through a direct interaction between the DAT and $\gamma$-synuclein, which disrupts the interaction between the DAT and $\alpha$-synuclein (Moszczynska et al., 2007). By disrupting the $\mathrm{DAT} / \alpha$-synuclein interaction, $\gamma$-synuclein not only reduces the level of dopamine reuptake, but consequentially mitigates the toxicity of exogenous dopamine in the presence of $\alpha$-synuclein. Moreover, dopamine has been reported to lead to the inactivation of $\gamma$-synuclein (LaVoie et al., 2005), therefore having increased DAT expression can lead to increased intracellular dopamine levels and subsequent inactivation of $\gamma$-synuclein.

Dopamine (also L-DOPA and other catecholamines) may inhibit fibrillation of $\alpha$-synuclein and amyloid $\beta$-peptide; their oxidation products are potent inhibitors of $\alpha$-synuclein fibrillation and may dissolve preformed fibrils (Conway et al., 2001; Li et al., 2002). The inhibitory activity of dopamine was attributed to the formation of covalent adducts between the o-quinone derivative of dopamine and $\alpha$-synuclein, causing an accumulation of the covalently modified protofibrils that are unable to fibrillate (Conway et al., 2001; Cappai et al., 2005). Both $\alpha$ - and $\gamma$-synucleins may form fibrils (Uversky et al., 2002), although this has not been observed in cocaine-treated animals or human patients. The addition of either $\gamma$-synuclein in a $1: 1$ molar ratio to $\alpha$-synuclein solution substantially increases the duration of the lag-time and reduces the elongation rate of $\alpha$-synuclein fibrillation (Uversky et al., 2002). Fibrillation is completely inhibited at a $4: 1$ molar excess of $\gamma$-synuclein over $\alpha$-synuclein (Hashimoto et al., 2001; Uversky et al., 2002), suggesting that $\gamma$-synucleins may act as a regulator of $\alpha$-synuclein fibrillation in vivo, potentially acting as chaperone (Uversky et al., 2002). Further molecular studies will be needed to establish the very function of the two synucleins in cocaine paradigms, in relation to DAT expression.

\section{Acknowledgements}

This study was supported by Swiss National Foundation grants 3100-059350 and 3100AO-100686 (J.L.D.). The authors are very grateful to Mrs V. DerocheGamonet (Institute for Neurosciences, Inserm538, Bordeaux) for scientific support and for critical comments and reading of the manuscript, and Mrs C. Deforel-Poncet for skilful assistance.

\section{Abbreviations}

$\mathrm{Ct}$, threshold cycle; DAT, dopamine transporter; GAPDH, glyceraldehyde-3phosphate dehydrogenase; GFP, green fluorescent protein; HEK293T, human embryonic kidney $293 \mathrm{~T}$ cells; LV- $\gamma$ Syn, lentivirus- $\gamma$-synuclein; LV- $\gamma$ SynsiRNAs, lentiviruses- $\gamma$-synuclein-siRNAs; LV-DAT, lentivirus-DAT; LV-siR NAs-DAT, lentiviruses DAT-siRNAs; NAc, nucleus accumbens; PBS, phosphate-buffered saline; PCR, polymerase chain reaction; VTA, ventral tegmental area.

\section{References}

Abeliovich, A., Schmitz, Y., Farinas, I., Choy-Lundberg, D., Ho, W.H., Castillo, P.E., Shinsky, N., Verdugo, J.M., Armanini, M., Ryan, A., Hynes, M., Phillips, H., Sulzer, D. \& Rosenthal, A. (2000) Mice lacking alphasynuclein display functional deficits in the nigrostriatal dopamine system. Neuron, 25, 239-252.

Aguilar, R.C. \& Wendland, B. (2003) Ubiquitin: not just for proteasomes anymore. Curr. Opin. Cell Biol., 15, 184-190.

Bahi, A., Boyer, F., Bussard, G. \& Dreyer, J.L. (2005) Silencing dopamine D3-receptors in the nucleus accumbens shell in vivo induces changes in cocaine-induced hyperlocomotion. Eur J. Neurosci., 21, 3415-3426.

Bahi, A., Boyer, F., Gumy, C., Kafri, T. \& Dreyer, J.L. (2004a) In vivo gene delivery of urokinase-type plasminogen activator with regulatable lentivirus induces behavioral changes in chronic cocaine administration. Eur J. Neurosci., 20, 3473-3488.

Bahi, A., Boyer, F., Kafri, T. \& Dreyer, J.L. (2004b) CD81-induced behavioral changes during chronic cocaine administration: in vivo gene delivery with regulatable lentivirus. Eur J. Neurosci., 19, 1621-1633.

Boyer, F. \& Dreyer, J.L. (2007) Alpha-synuclein in the nucleus accumbens induces changes in cocaine behavior in rats. Eur J. Neurosci., 26, 2764 2776.

Brenz-Verca, M.S., Bahi, A., Boyer, F., Wagner, G.C. \& Dreyer, J.L. (2003) Distribution of alpha- and gamma-synucleins in the adult rat brain and their modification by high-dose cocaine treatment. Eur. J. Neurosci., 18, 19231938. 
Buchman, V.L., Hunter, H.J., Pinon, L.G., Thompson, J., Privalova, E.M., Ninkina, N.N. \& Davies, A.M. (1998) Persyn, a member of the synuclein family, has a distinct pattern of expression in the developing nervous system. J. Neurosci., 18, 9335-9341.

Cappai, R., Leck, S.L., Tew, D.J., Williamson, N.A., Smith, D.P., Galatis, D., Sharples, R.A., Curtain, C.C., Ali, F.E., Cherny, R.A., Culvenor, J.G., Bottomley, S.P., Masters, C.L., Barnham, K.J., Hill, A.F. (2005) Dopamine promotes alpha-synuclein aggregation into SDS-resistant soluble oligomers via a distinct folding pathway. FASEB J., 19, 1377-1379.

Chen, N. \& Reith, M.E. (2000) Structure and function of the dopamine transporter. Eur. J. Pharmacol., 405, 329-339.

Choi, P., Golts, N., Snyder, H., Chong, M., Petrucelli, L. \& Hardy, J. (2001) Co association of parkin and alpha-synuclein. Neuroreport, 12, 2839-2843.

Chung, K.K., Zhang, Y., Lim, K.L., Tanaka, Y., Huang, H., Gao, J., Ross, C.A., Dawson, V.L. \& Dawson, T.M. (2001) Parkin ubiquitinates the alphasynuclein-interacting protein, synphilin-1: implications for Lewy-body formation in Parkinson disease. Nat. Med., 7, 1144-1150.

Conway, K.A., Rochet, J.C., Bieganski, R.M. \& Lansbury, P.T. Jr (2001) Kinetic stabilization of the alpha-synuclein protofibril by a dopamine-alphasynuclein adduct. Science, 294, 1346-1349.

Daniels, G.M. \& Amara, S.G. (1999) Regulated trafficking of the human dopamine transporter. Clathrin-mediated internalization and lysosomal degradation in response to phorbol esters. J. Biol. Chem., 274, 35794-35801.

Ferguson, S.S. (2003) Receptor tyrosine kinase transactivation: fine-tuning synaptic transmission. Trends Neurosci., 26, 119-122.

Galvin, J.E., Giasson, B., Hurtig, H.I., Lee, V.M. \& Trojanowski, J.Q. (2000) Neurodegeneration with brain iron accumulation, type-1 is characterized by alpha-, beta-, and gamma-synuclein neuropathology. Am. J. Pathol., 157, 361-368.

Galvin, J.E., Uryu, K., Lee, V.M. \& Trojanowski, J.Q. (1999) Axon pathology in Parkinson's disease and Lewy body dementia hippocampus contains alpha-, beta-, and gamma-synuclein. Proc. Natl Acad. Sci. USA, 96, 1345013455 .

George, J.M. (2001) The synucleins. Gen. Biol., 3, 3002.1-3002.6 [Review].

Hashimoto, M., Rockenstein, E., Mante, M., Mallory, M. \& Masliah, E. (2001) Beta-synuclein inhibits alpha-synuclein aggregation: a possible role as an anti-parkinsonian factor. Neuron, 32, 213-223.

Hicke, L. (2001) Protein regulation by mono-ubiquitin. Nat. Rev. Mol. Cell Biol., 2, 195-201.

Hicke, L. \& Dunn, R. (2003) Regulation of membrane protein transport by ubiquitin and ubiquitin-binding proteins. Ann. Rev. Cell Dev. Biology, 19, $141-172$.

Horn, A.S. (1990) Dopamine uptake: a review of progress in the last decade. Prog. Neurobiol., 34, 387-400.

Jia, T., Liu, Y.E., Liu, J. \& Shi, Y.E. (1999) Stimulation of breast cancer invasion and metastasis by synuclein gamma. Cancer Res., 59, 742-747.

Jiang, H., Ren, Y., Zhao, J. \& Feng, J. (2004) Parkin protects human dopaminergic neuroblastoma cells against dopamineinduced apoptosis. Hum. Mol. Genet., 13, 1745-1754.

Kim, T.D., Paik, S.R. \& Yang, C.H. (2002) Structural and functional implications of C-terminal regions of alpha-synuclein. Biochemistry., 41 $13782-13790$

Kuhn, M., Haebig, K., Bonin, M., Ninkina, N., Buchman, V.L., Poths, S. \& Riess, O. (2007) Whole genome expression analyses of single- and doubleknock-out mice implicate partially overlapping functions of alpha- and gamma-synuclein. Neurogenetics, $\mathbf{8}, 71-81$.

Lavedan, C. (1998) The synuclein family. Gen. Res., 8, 871-880.

LaVoie, M.J., Ostaszewski, B.L., Weihofen, A., Schlossmacher, M.G. \& Selkoe, D.J. (2005) Dopamine covalently modifies and functionally inactivates parkin. Nat. Med., 11, 1214-1221.

Li, J.Y., Henning-Jensen, P. \& Dahlstrom, A. (2002) Differential localization of alpha, beta- and gamma-synucleins in the rat CNS. Neuroscience, 113, 463478

Liang, T., Spence, J., Liu, L., Strother, W.N., Chang, H.W., Ellison, J.A., Lumeng, L., Li, T.K., Foroud, T. \& Carr, L.G. (2003) alpha-Synuclein maps to a quantitative trait locus for alcohol preference and is differentially expressed in alcoholpreferring and - nonpreferring rats. Proc. Natl Acad. Sci. USA, 100, 4690-4695.

Lim, K.L., Chew, K.C., Tan, J.M., Wang, C., Chung, K.K., Zhang, Y., Tanaka, Y., Smith, W., Engelender, S., Ross, C.A., Dawson, V.L. \& Dawson, T.M. (2005) Parkin mediates nonclassical, proteasomalindependent ubiquitination of synphilin-1: implications for Lewy body formation. J. Neurosci., 25, 2002-2009.

LoBianco, C., Ridet, J.L., Schneider, B.L., Deglon, N. \& Aebischer, P. (2002) Alpha-synucleinopathy and selective dopaminergic neuron loss in a rat lentiviral-based model of Parkinson's disease. Proc. Natl Acad. Sci. USA, 99, 10813-10818.

Maggos, C.E., Spangler, R., Zhou, Y., Schlussman, S.D., Ho, A. \& Kreek, M.J. (1998) Quantitation of dopamine transporter mrna in the rat brain: mapping, effects of binge cocaine administration and withdrawal. Synapse, 26, 55-61.

Mash, D.C., Ouyang, Q., Pablo, J., Basile, M., Izenwasser, S., Lieberman, A. \& Perrin, R.J. (2003) Cocaine abusers have an overexpression of alphasynuclein in dopamine neurons. J. Neurosci., 23, 2564-2571.

Maurage, C.A., Ruchoux, M.M., de Vos, R., Surguchov, A. \& Destee, A. (2003) Retinal involvement in dementia with Lewy bodies: a clue to hallucinations? Ann. Neurol., 54, 542-547.

Melikian, H.E. \& Buckley, K.M. (1999) Membrane trafficking regulates the activity of the human dopamine transporter. J. Neurosci., 19, 7699-7710.

Mosharov, E.V., Staal, R.G., Bove, J., Prou, D., Hananiya, A., Markov, D., Poulsen, N., Larsen, K.E., Moore, C.M., Troyer, M.D., Edwards, R.H., Przedborski, S. \& Sulzer, D. (2006) Alpha-synuclein overexpression increases cytosolic catecholamine concentration. J. Neurosci., 26, 93049311

Moszczynska, A., Saleh, J., Zhang, H., Vukusic, B., Lee, F.J.S. \& Liu, F. (2007) Parkin disrupts the alpha-synuclein/dopamine transporter interaction: consequences toward dopamine-induced toxicity. J. Mol. Neurosci., 32, $217-$ 227.

Murphy, D.D., Rueter, S.M., Trojanowski, J.Q. \& Lee, V.M. (2000) Synucleins are developmentally expressed, and alpha-synuclein regulates the size of the presynaptic vesicular pool in primary hippocampal neurons. J. Neurosci., 20, 3214-3220.

Oluwatosin-Chigbu, Y., Robbins, A., Scott, C.W., Arriza, J.L., Reid, J.D. \& Zysk, J.R. (2003) Parkin suppresses wild-type alphasynuclein-induced toxicity in SHSY-5Y cells. Biochem. Biophys. Res. Commun., 309, 679-684.

Paxinos, G. \& Watson, C. (1998) The Rat Brain in Stereotactic Coordinates. Academic, New York.

Perez, R.G., Waymire, J.C., Lin, E., Liu, J.J., Guo, F. \& Zigmond, M.J. (2002) A role for alpha-synuclein in the regulation of dopamine biosynthesis. J. Neurosci., 22, 3090-3099.

Petrucelli, L., O'Farrell, C., Lockhart, P.J., Baptista, M., Kehoe, K., Vink, L., Choi, P., Wolozin, B., Farrer, M., Hardy, J. \& Cookson, M.R. (2002) Parkin protects against the toxicity associated with mutant alpha-synuclein: proteasome dysfunction selectively affects catecholaminergic neurons. Neuron, 36, 1007-1019.

Pickart, C.M. (2000) Ubiquitin in chains. Trends Biochem. Sci., 25, 544-548. Pinhasov, A., Ilyin, S.E., Crooke, J., Amato, F.A., Vaidya, A.H., Rosenthal, D., Brenneman, D.E. \& Malatynska, E. (2005) Different levels of gammasynuclein mRNA in the cerebral cortex of dominant, neutral and submissive rats selected in the competition test. Genes, Brain Behav., 4, 60-64.

Pristupa, Z.B., McConkey, F., Liu, F., Man, H.Y., Lee, F.J., Wang, Y.T. \& Niznik, H.B. (1998) Protein kinase-mediated bidirectional trafficking and functional regulation of the human dopamine transporter. Synapse, 30, 79-87.

Qin, A., Ouyang, Q., Pablo, J. \& Mash, D.C. (2005) Cocaine abuse elevates alpha-synuclein and dopamine transporter levels in human striatum. Neuroreport, 16, 1489-1493.

Shimura, H., Schlossmacher, M.G., Hattori, N., Frosch, M.P., Trockenbacher, A., Schneider, R., Mizuno, Y., Kosik, K.S. \& Selkoe, D.J. (2001) Ubiquitination of a new form of alpha-synuclein by parkin from human brain: implications for Parkinson's disease. Science, 293, 263-269.

Sidhu, A., Wersinger, C. \& Vernier, P. (2004a) alpha-Synuclein regulation of the dopaminergic transporter: a possible role in the pathogenesis of Parkinson's disease. FEBS Lett., 565, 1-5.

Sidhu, A., Wersinger, C., Vernier, P. (2004b) Does alpha-synuclein modulate dopaminergic synaptic content and tone at the synapse? FASEB J., 18, 637647.

Surgucheva, I., McMahan, B., Ahmed, F., Tomarev, S., Wax, M.B. \& Surguchov, A. (2002) Synucleins in glaucoma: implication of gammasynuclein in glaucomatous alterations in the optic nerve. J. Neurosci. Res., 68, 97-106.

Surgucheva, I.G., Sivak, J.M., Fini, M.E., Palazzo, R.E. \& Surguchov, A.P. (2003) Effect of gamma-synuclein overexpression on matrix metalloproteinases in retinoblastoma Y79 cells. Arch. Biochem. Biophys., 410, 167-176.

Surguchov, A., McMahan, B., Masliah, E. \& Surgucheva, I. (2001b) Synucleins in ocular tissues. J. Neurosci. Res., 65, 68-77.

Surguchov, A., Palazzo, R.E. \& Surgucheva, I. (2001a) Gamma synuclein: subcellular localization in neuronal and non-neuronal cells and effect on signal transduction. Cell Motil Cytoskeleton, 49, 218-228.

Uversky, Z.N., Li, J., Souillac, P., Millett, I.S., Doniach, S., Jakes, R., Goedert, M. \& Fink, A.L. (2002) Biophysical properties of the synucleins and their 
propensities to fibrillate: inhibition of alpha-synuclein assembly by beta- and gamma-synucleins. J. Biol. Chem., 277, 11970-11978.

Wang, Y.L., Takeda, A., Osaka, H., Hara, Y., Furuta, A., Setsuie, R., Sun, Y.J., Kwon, J., Sato, Y., Sakurai, M., Noda, M., Yoshikawa, Y. \& Wada, K. (2004a) Accumulation of beta- and gamma-synucleins in the ubiquitin carboxyl-terminal hydrolase L1-deficient gad mouse. Brain Res., 1019, 1-9.

Wersinger, C., Prou, D., Vernier, P. \& Sidhu, A. (2003a) Modulation of dopamine transporter function by alpha-synuclein is altered by impairment of cell adhesion and by induction of oxidative stress. FASEB J., 17, 21512153.

Wersinger, C., Prou, D., Vernier, P., Niznik, H.B. \& Sidhu, A. (2003b) Mutations in the lipid-binding domain of alpha-synuclein confer overlapping, yet distinct, functional properties in the regulation of dopamine transporter activity. Mol. Cell Neurosci., 24, 91-105.
Wersinger, C., Rusnak, M. \& Sidhu, A. (2006) Modulation of the trafficking of the human serotonin transporter by human alpha-synuclein. Eur. J. Neurosci., 24, 55-64.

Wersinger, C. \& Sidhu, A. (2003) Attenuation of dopamine transporter activity by alpha-synuclein. Neurosci. Lett., 340, 189-192.

Wersinger, C. \& Sidhu, A. (2005) Disruption of the interaction of alphasynuclein with microtubules enhances cell surface recruitment of the dopamine transporter. Biochemistry, 44, 13612-13624.

Xu, J., Kao, S.Y., Lee, F.J., Song, W., Jin, L.W. \& Yankner, B.A. (2002) Dopamine-dependent neurotoxicity of alpha-synuclein: a mechanism for selective neurodegeneration in Parkinson disease. Nat. Med., 8, 600-606. Yamada, M., Mizuno, Y. \& Mochizuki, H. (2005) Parkin gene therapy for alpha-synucleinopathy: a rat model of Parkinson's disease. Human Gene Ther. 16, 262-270. 


\section{Appendix S1.}

In vitro assay of lentivirus-mediated $\gamma$-synuclein expression in HEK 293T cells: $\gamma$ Synuclein was cloned into a tetracycline-regulatable Lentivirus system (see Methods). The efficiency to LV- $\gamma$ Syn to mediated $\gamma$-synuclein mRNA overexpression was check in vitro (see methods). HEK 293 T cells were infected with different concentrations of virus (1, 2, 4 and $8 \mu \mathrm{l}$ ) and the levels of $\gamma$-synuclein mRNA were measured by qRT-PCR. Transcripts levels were normalized against cyclophilin $\mathrm{F}$ as an endogenous control and GAPDH mRNA was also quantified. As shown in Figure S1A, in absence of doxycycline expression of $\gamma$-synuclein was dose-dependent displaying a 2 fold, 4.2 fold and 9.7 fold increase with $2,4 \mu \mathrm{l}$ and $8 \mu \mathrm{l}$ viral stock $\left(p<0.001, F_{(4,40)}=73.7\right)$. In presence of doxycycline $(30 \mathrm{ng} / \mathrm{ml}), \mathrm{LV}$ $\gamma$ Syn-mediated $\gamma$-synuclein expression was totally abolished, and $\gamma$-synuclein mRNA returned to basal levels (Figure S1A). GAPDH mRNA was not affected by these conditions showing a constant ratio of 1.3 .

In order to knock-down $\gamma$-synuclein expression, lentivirus-based RNA interference was used. Three different siRNAs were designed, targeted against three different regions of the $\gamma$-synuclein mRNA and cloned under a U6 promoter into the transfer plasmid of the lentivirus system. These lentivirus constructs were not doxycycline-regulable. The three LV- $\gamma$ Syn-siRNAs were mixed at equal amounts for optimal inhibition of $\gamma$-synuclein expression, and this mix was tested in HEK293T cells overexpressing $\gamma$-synuclein.

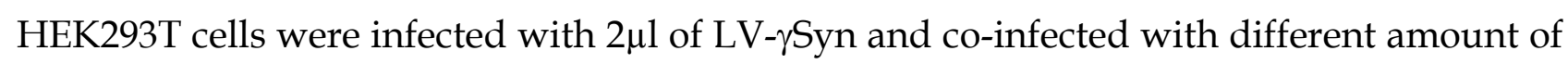
the mix of LV- $\gamma$ Syn-siRNAs $(0.5,1,2$, and $4 \mu \mathrm{l})$ and $\gamma$-synuclein expression was measured by qRT-PCR (Figure 1B). This resulted in drastic down-regulation of $\gamma$-synuclein, yielding residual $\gamma$-synuclein mRNA levels of 46, 24, 9 and 1.6\% respectively (Figure S1B, $p<0.001$; $\left.F_{(5,24)}=101.04\right)$. The expression levels of endogenous control (cyclophilin F and GAPDH) were not affected by LV- $\gamma$ Syn-siRNAs.

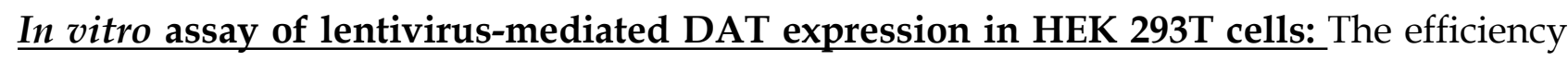
of the LV-DAT to drive DAT overexpression was also assessed in vitro. HEK293T cells were infected using 2, 4 or $8 \mu$ l of the LV-DAT stock, and the DAT mRNA was quantified by qRT-PCR. Cyclophilin F and GAPDH were used as endogenous control. In absence of doxycycline, LV-DAT infection in HEK293T cells led to titre-dependent overexpression of DAT mRNA (Figure 1C), yielding a 3.5, 5.2 and 9.6 fold increase of DAT mRNA upon 
infection with 2, 4 and $8 \mu$ of LV-DAT, respectively $\left(p<0.001, F_{(3,32)}=64.99\right)$. GAPDH mRNA levels were unchanged in these conditions, displaying a constant ratio of 1.3. In presence of doxycycline, DAT mRNA increases were totally abolished, returning back to basal levels, but GAPDH mRNA levels were unaffected (Figure 1C).

As for $\gamma$-synuclein, knock-down of DAT expression was assessed using LV-DAT-siRNAs. For this purpose, three different siRNAs targets against the DAT mRNA were designed, cloned into the lentivirus system under a U6 promoter. The three LV-DAT-siRNAs were mixed at an equal amount for optimal DAT knock-down and tested. HEK293T cells were co-infected with $2 \mu 1$ of LV-DAT and different amount of mix of LV-DAT-siRNAs $(0.5,1,2$ and $4 \mu \mathrm{l}$ ). Infection with $1 \mu \mathrm{l}$ mix of LV-DAT-siRNAs induces a $57 \%$ down-regulation of DAT expression (Figure 1D, $p<0.0001 ; F_{(5 ; 24)}=56.05$ ), whereas infection with $2 \mu 1$ and $4 \mu 1$ mix of LV-DAT-siRNAs leads to $25 \%$ and $9 \%$ residual DAT expression levels, respectively (Figure 1D, $\left.p<0.0001 ; F_{(5 ; 24)}=56.05\right)$. The expression levels of endogenous control (cyclophilin F and GAPDH) were not affected by LV-DAT-siRNAs.

In vitro evaluation of target specificity of LV-siRNAs: $\gamma$-Synuclein is a small protein and displays ca.95\% homology to $\alpha$-synculein. In order to test for the specificity of our LV$\gamma$ Syn-siRNA, HEK293T cells have been transfected with different closely related contructs. Transfection of HEK293T cells with LV- $\gamma$ Syn-siRNA has no effect on expression of $\alpha$ synuclein, and vice-versa transfection of LV- $\alpha$ Syn-siRNA do not affect $\gamma$-synuclein expression, despite of the very high homology of the two genes (Fig.S2 and Table S1). Other LV-siRNA, eg. LV-GFP-siRNA, also did not affect expression of $\gamma$-synuclein. 


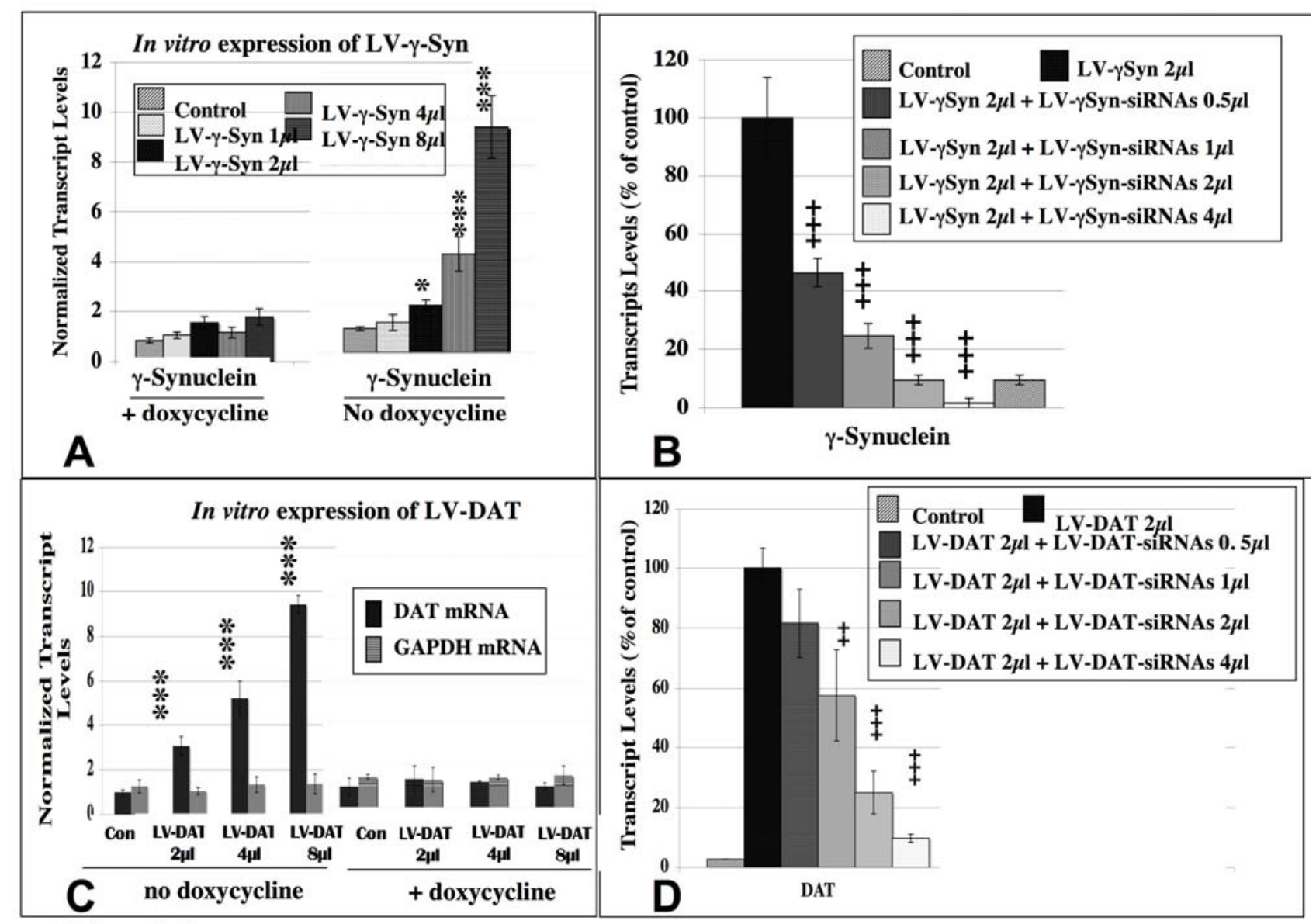

Fig S1

Figure S1.

In vitro Expression of Lentivirus Constructs in HEK-293T: HEK-293T cells were infected as indicated. After 72h, Total RNA was extracted using the Trizol method, cDNA was prepared and transcripts levels were quantified using real-time PCR method with specific primers. Control: Non-infected HEK 293 T cells. Values indicate means \pm SEM.

S1A: In vitro expression of $\gamma$-synuclein of HEK293T cells transfected with LV- $\gamma$ Syn. HEK $293 \mathrm{~T}$ cells were infected with $0,1,2,4$, or $8 \mu 1$ of $L V-\gamma$ Syn in culture medium in the presence or absence of doxycycline (30ng/ml) as indicated. ${ }^{*} p<0.05,{ }^{* * *} p<0.001$ compared to control cells (non infected)

S1B: in vitro evaluation of LV- $\gamma$ Syn-siRNAs efficiency in HEK 293 T cells. HEK-293T cells were infected with $2 \mu 1$ of LV- $\gamma$ Syn alone or ci-infected with $0.5,1,2$, or $4 \mu 1$ LV- $\gamma$ Syn-siRNAs. ${ }^{+++} p<0.001$ compared to LV- $\gamma$ Syn infected cells. 
S1C: in vitro expression of DAT mRNA using LV-DAT in HEK 293T cells. HEK 293T cells were infected with $0,2,4$, or $8 \mu l$ of LV-DAT in culture medium in the presence or absence of doxycycline $(30 \mathrm{ng} / \mathrm{ml})$ as indicated. ${ }^{* * *} p<0.001$ compared to control cells (non infected) S1D: in vitro evaluation of LV-DAT-siRNAs efficiency in HEK-293T cells. HEK-293T cells were infected with $2 \mu \mathrm{l}$ of LV-DAT alone or together with $0.5,1,2$, or $4 \mu \mathrm{l}$ of LV- DATsiRNAs. ${ }^{++} p<0.01,{ }^{+++} p<0.001$ compared to LV-DAT infected cells. 
Total Locomotor Activity Total Locomotor Activity Session A (no doxycycline) Session B (+ doxycycline)
Total Locomotor Activity

Session C (no doxycycline)

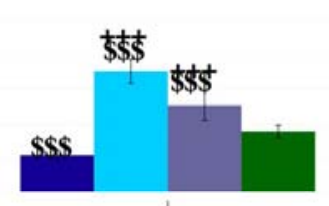

LV-GFP

LV-DAT
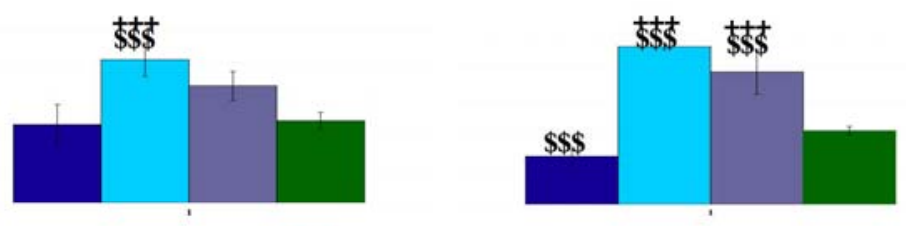

LV-DAT -siRNAs

LV-DAT + LV-DAT -siRNAs

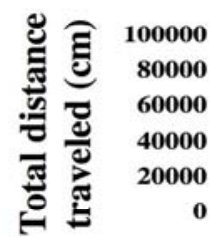

Fig S2B
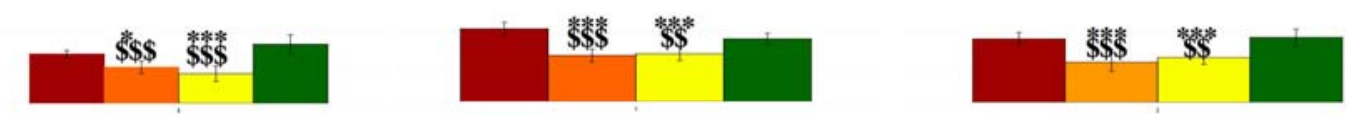

LV- $\gamma$-Syn + LV- $\gamma$-Syn -siRNAs LV- $\gamma$-Syn -siRNAs

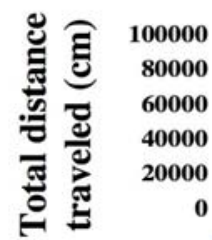

Fig S2C
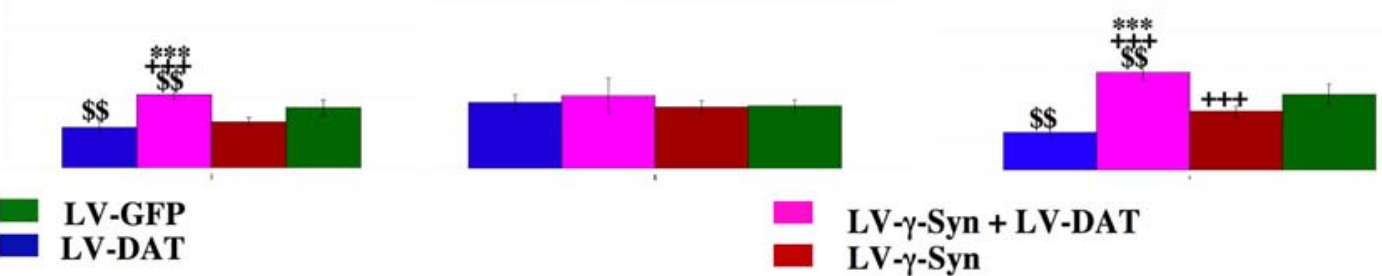

Figure S2.

In vitro Evaluation of Target Specificity of LV- $\gamma$ Syn-siRNA:

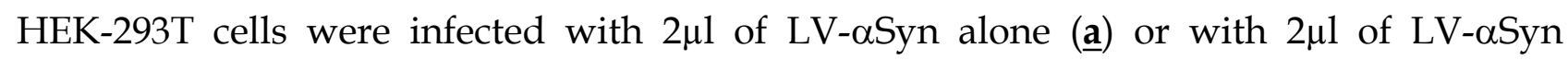
together with either $2 \mu \mathrm{l}$ of LV- $\alpha$ Syn-siRNAs $(\underline{\mathbf{b}})$ or with $2 \mu \mathrm{LV}-\alpha \operatorname{Syn}-\operatorname{siRNAs}(\underline{\mathbf{c}})$. Also cells were infected with $2 \mu \mathrm{LV}-\gamma \operatorname{Syn}$ alone $(\underline{\mathbf{d}})$ or with with $2 \mu \mathrm{l}$ of LV- $\gamma$ Syn together with either

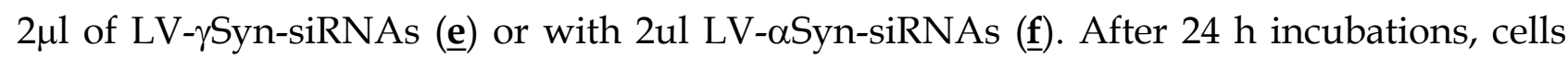
were harvested and mRNA extracted and measured by qRT-PCR. Figure S2A: evaluation of $\gamma$-synuclein mRNA; Figure S2B: evaluation of $\alpha$-synuclein mRNA. See also Table S1 for details. ${ }^{++} p<0.01,{ }^{+++} p<0.001$ compared to LV-DAT infected cells. 


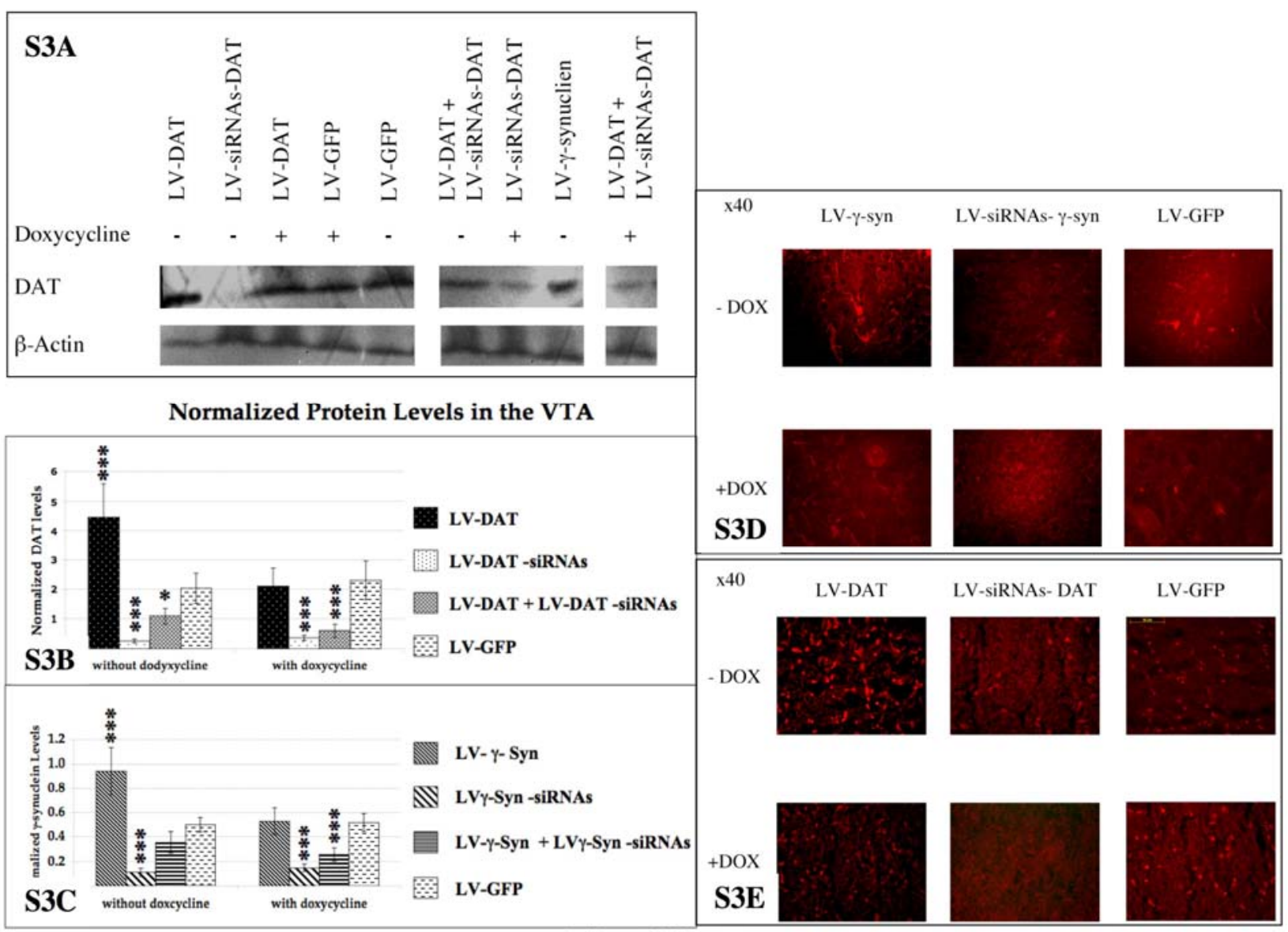

Fig S3

Figure S3.

Locomotor Activity upon DAT and $\gamma$-Synuclein Overexpression or Silencing in the

Nacc. Total distance travelled in locomotor activity from figures 1 and 2 . Each bar represents the average of 8 animals recorded during 5 consecutive days. Bars represent means \pm SEM. 


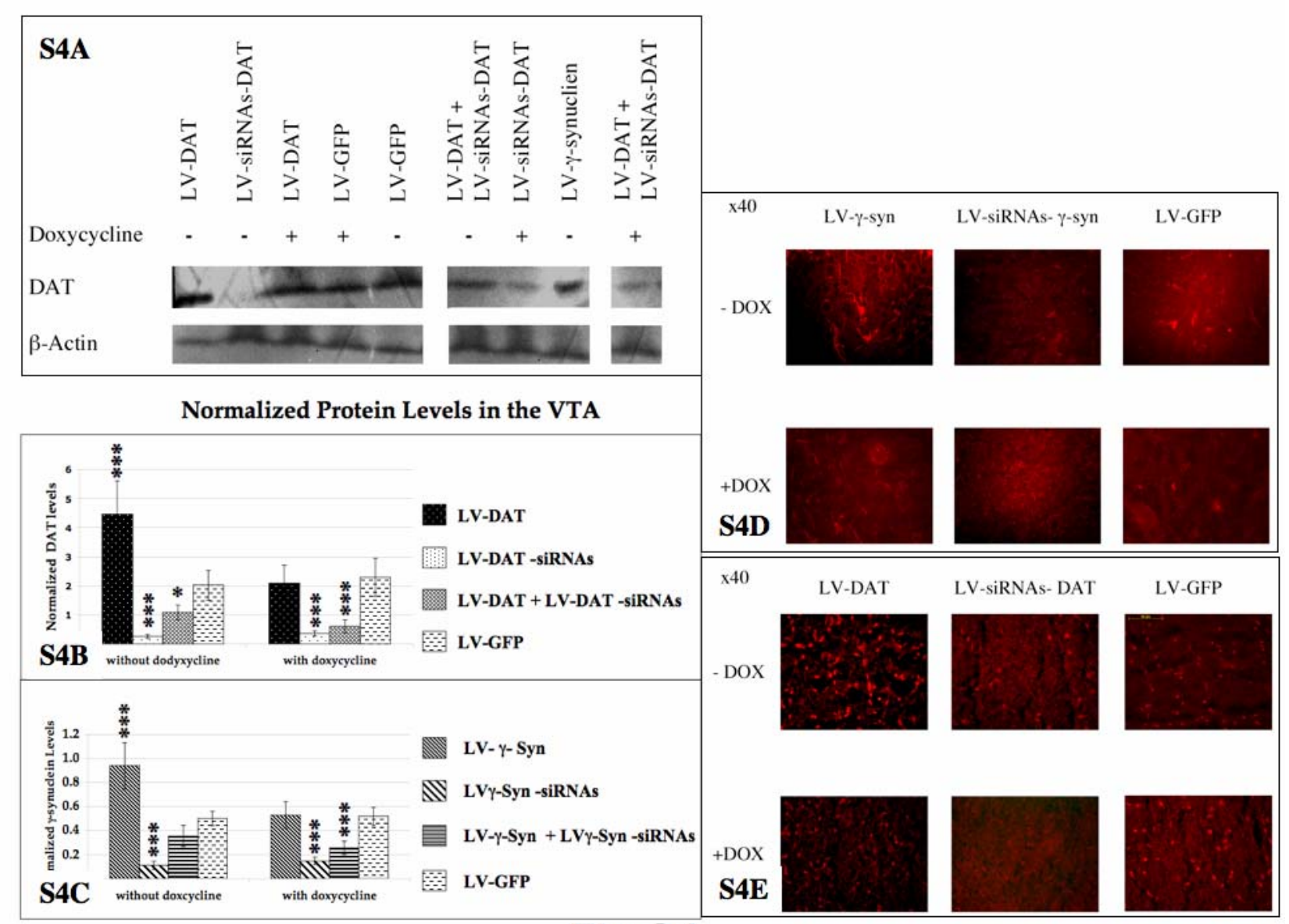

Fig S4

Figure S4.

Expression of DAT and $\gamma$-Synuclein in the VTA upon lentivirus injection in the NAc.

S4A: Western Blot analysis were performed as in Fig. 4 from VTA samples of animals treated with lentivirus injected in the NAc._Western blots from gels loaded with $20 \mu \mathrm{g}$ protein/lane were analyzed for either DAT or $\gamma$ synuclein expression, and reprobed with $\beta$ actin antibody to compare DAT or $\gamma$ synuclein expression in the VTA upon lentivirus infection in the NAc.

DAT and $\gamma$-Synuclein signals were estimated as in Fig. 4, using multi-anlyst software and normalized against $\beta$-actin signal (see Fig. 4 and Methods Section for details). Data represents means \pm SEM. S4B: In vivo DAT protein levels quantification in the VTA. ${ }^{* * *} p<0.001,{ }^{* *} p<0.01$ : semi-quantitative DAT protein quantification vs. DAT quantification detected from LV-GFP injected rats S4C: in vivo $\gamma$-synuclein protein levels quantification in the VTA. ${ }^{* *} p<0.001,{ }^{* *} p<0.01$ : semi-quantitative $\gamma$-synuclein protein quantification vs. $\gamma$ synuclein quantification detected from LV-GFP injected rats. 
S4D: High magnification immunohistochemistry of $\gamma$-synuclein expression in the VTA. Immunohistochemistry was performed after in vivo delivery of LV- $\gamma$ Syn $(2 \mu 1)$ combined with LV-DAT $(2 \mu \mathrm{l})$ (left panels) or LV-siRNA-g-syn (middle panels) or LV-GFP (right panels). Lentiviruses injections were performed into the Nac according to the coordinates described in the Methods section. Animals used for behavioral analysis were fed either $5 \%$ sucrose or $5 \%$ sucrose $/ 0.02 \%$ doxycycline (see text for details). Animals were killed at the end of behavioral analyses; brains were dissected out, and processed for immunohistochemistry (see Methods). Brain sections from animals fed 5\% sucrose (upper panels) or doxycycline (lower panels) were labeled using antibodies against $\gamma$-synuclein and were visualized by fluorescence microscopy at 40X magnitude.

S4E: High magnification immunohistochemistry of DAT expression in the VTA. Injections of LV- $\gamma$ Syn $(2 \mu \mathrm{l})$ combined with LV-DAT $(2 \mu \mathrm{l})$ (left panels) or LV-siRNA-DAT (middle panels) or LV-GFP (right panels) were performed in the Nac, and tissues processed as described above. 


\section{Table S1.}

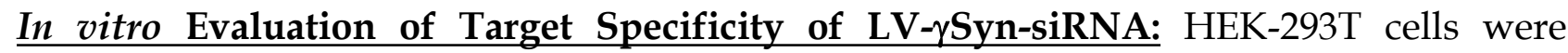

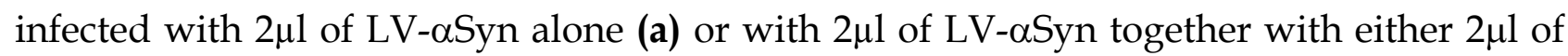

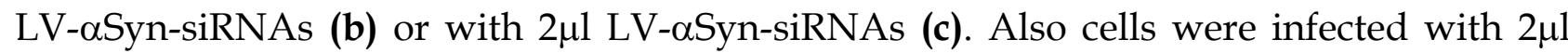
LV- $\gamma$ Syn alone (d) or with with $2 \mu 1$ of LV- $\gamma$ Syn together with either $2 \mu l$ of LV- $\gamma$ Syn-siRNAs (e) or with 2 ul LV- $\alpha$ Syn-siRNAs (f). After $24 \mathrm{~h}$ incubations, cells were harvested, mRNA was extracted and measured by qRT-PCR, by evaluation of $\gamma$-synuclein and of $\alpha$-synuclein mRNA, against GAPDH as standard. See also Figure S2 for details.

\begin{tabular}{|c|c|c|c|c|c|}
\hline $\mathbf{a}$ & \multicolumn{3}{|c|}{ LV- $\alpha$-synuclein } & \multirow{2}{*}{$\begin{array}{c}\text { Average } \\
1.813\end{array}$} & \multirow{2}{*}{$\frac{\text { STD }}{0.423}$} \\
\hline$\alpha$ synuclein & 1.516 & 1.625 & 2.297 & & \\
\hline GAPDH & 0.287 & 0.018 & 0.117 & 0.141 & 0.136 \\
\hline$\gamma$ synuclein & 0.001 & 0 & 0 & 0.000 & 0.001 \\
\hline b & \multicolumn{3}{|c|}{ LV- $\alpha$-synuclein+ LV-siRNAs- $\alpha$-synuclein } & & \\
\hline$\alpha$ synuclein & 0.189 & 0.134 & 0.287 & 0.203 & 0.078 \\
\hline GAPDH & 0.379 & 0.047 & 0.088 & 0.171 & 0.181 \\
\hline$\gamma$ synuclein & 0 & 0 & 0 & 0.000 & 0.000 \\
\hline C & \multicolumn{3}{|c|}{ LV- $\alpha$-synuclein+ LV-siRNAs- $\gamma$-synuclein } & & \\
\hline$\alpha$ synuclein & 1.149 & 2.462 & 2 & 1.870 & 0.666 \\
\hline GAPDH & 0.189 & 0.144 & 0.203 & 0.179 & 0.031 \\
\hline$\gamma$ synuclein & 0.005 & 0.033 & 0 & 0.013 & 0.018 \\
\hline d & \multicolumn{3}{|c|}{ LV- $\gamma$-synuclein } & & \\
\hline$\alpha$ synuclein & 0.004 & 0.007 & 0.004 & 0.005 & 0.002 \\
\hline GAPDH & 0.233 & 0.077 & 0 & 0.103 & 0.119 \\
\hline$\gamma$ synuclein & 10.556 & 12.996 & 14.929 & 12.827 & 2.191 \\
\hline e & \multicolumn{3}{|c|}{ LV- $\gamma$-synuclein+ LV-siRNAs- $\gamma$-synuclein } & & \\
\hline$\alpha$ synuclein & 0.007 & 0.011 & 0.009 & 0.009 & 0.002 \\
\hline GAPDH & 0.435 & 0.33 & 0.406 & 0.390 & 0.054 \\
\hline$\gamma$ synuclein & 2 & 6.063 & 1.866 & 3.310 & 2.385 \\
\hline $\mathbf{f}$ & \multicolumn{3}{|c|}{ LV- $\gamma$-synuclein+ LV-siRNAs- $\alpha$-synuclein } & & \\
\hline$\alpha$ synuclein & 0.467 & 0.044 & 0.015 & 0.175 & 0.253 \\
\hline GAPDH & 0.01 & 0.233 & 0.203 & 0.149 & 0.121 \\
\hline$\gamma$ synuclein & 8 & 12.126 & 11.314 & 10.48 & 2.19 \\
\hline
\end{tabular}

